Research Article

\title{
Set-Valued SU-Type Fixed Point Theorems via Gauge Function with Applications
}

\author{
Amjad Ali, ${ }^{1}$ Monairah Alansari, ${ }^{2}$ Fahim Uddin $\left(D,{ }^{1}\right.$ Muhammad Arshad, ${ }^{1}$ Awais Asif, ${ }^{1}$ \\ and Ghada Ali Basendwah ${ }^{2}$ \\ ${ }^{1}$ Department of Mathematics and Statistics, International Islamic University, Islamabad, Pakistan \\ ${ }^{2}$ Department of Mathematics, King Abdulaziz University, P.O. Box 80203, Jeddah 21589, Saudi Arabia \\ Correspondence should be addressed to Fahim Uddin; fahim.uddin@iiu.edu.pk
}

Received 26 December 2020; Revised 21 March 2021; Accepted 25 March 2021; Published 19 May 2021

Academic Editor: Naeem Saleem

Copyright (C) 2021 Amjad Ali et al. This is an open access article distributed under the Creative Commons Attribution License, which permits unrestricted use, distribution, and reproduction in any medium, provided the original work is properly cited.

In this article, we have designed two existence of fixed point theorems which are regarding to set-valued SU-type $\theta_{\eta}$-contraction and $\Gamma_{\alpha}$-contraction via gauge function in the setting of metric spaces. An extensive set of nontrivial example will be given to justify our claim. At the end, we will give an application to prove the existence behavior for the system of functional equation in dynamical system and integral inclusion.

\section{Introduction and Preliminaries}

The most publicized famous result in nonlinear analysis is Banach contraction principle, which made clear a systematic rule to find the fixed point of a given mapping on a metric space. So far, numerous authors have studied this classical result to examine the existence and uniqueness of a solution for different forms of contractive structure.

In 2014, Jleli and Samet [1] introduced the concept of a new contraction known as the $\theta$-contraction, which generalizes the Banach contraction principle in a beautiful way.

In 2015, Khojasteh et al. [2] introduced simulation function. Recently, many researchers have proved fixed point theorems for Suzuki-type (SU) mappings in metric space (see $[3,4])$.

Let $(X, ð)$ be a metric space. For $\mu \in X$ and $\beta_{1} \subseteq X$, let $\mathrm{CL}(X)$ and $\mathrm{CB}(X)$ denote the family of all nonempty closed subsets and the family of all nonempty closed bounded subsets of $X$. Design the Pompeiu-Hausdorff metric $H_{d}$ induced by $\partial$ on $\mathrm{CB}(X)$ as

$$
H_{d}\left(\beta_{1}, \beta_{2}\right)=\max \left\{\sup _{\mu_{1} \in \beta_{1}} \text { ð }\left(\mu_{1}, \beta_{2}\right), \sup _{\mu_{2} \in \beta_{2}} \text { ð }\left(\mu_{2}, \beta_{1}\right)\right\},
$$

for all $\beta_{1}, \beta_{2} \in \mathrm{CL}(X)$ and $ð\left(\mu_{1}, \beta_{1}\right)=\inf \left\{ð\left(\mu_{1}, \mu_{2}\right)\right.$ : $\left.\mu_{2} \in \beta_{1}\right\}$. A point $\mu \in X$ is said to be a fixed point of
$T: X \longrightarrow \mathrm{CB}(X)$, if $\mu \in T(\mu)$. If, for $\mu_{0} \in X$, there exists a sequence $\left\{\mu_{l}\right\}$ in $X$ such that $\mu_{l} \in T\left(\mu_{l-1}\right)$, then $O\left(T, \mu_{0}\right)=$ $\left\{\mu_{0}, \mu_{1}, \mu_{2}, \ldots\right\}$ is said to be an orbit of $T: X \longrightarrow \mathrm{CB}(X)$. Mapping $f: X \longrightarrow \mathbb{R}$ is said to be $T$-orbitally lower semicontinuous (o.l.s.c), if a sequence $\left\{\mu_{l}\right\}$ in $O\left(T, \mu_{0}\right)$ and $\left.\mu_{\iota} \longrightarrow \varrho \Rightarrow f(\rangle\right) \leq \liminf _{\iota} \mathrm{f}\left(\mu_{l}\right)$

A multivalued mapping $T: X \longrightarrow \mathrm{CB}(X)$ is called a Nadler-contraction, if there exists $\gamma \in(0,1)$ such that

$$
H_{d}\left(T\left(\mu_{1}\right), T\left(\mu_{2}\right)\right) \leq \gamma ð\left(\mu_{1}, \mu_{2}\right) \quad \text { for all } \mu_{1}, \mu_{2} \in X \text {. }
$$

Nadler [5] obtained the multivalued version of the Banach contraction principle. Let $(X, ð)$ be a complete metric space and $T: X \longrightarrow \mathrm{CL}(X)$ be a Nadler-contraction. Then, $T$ has a fixed point. Recently, Vetro [6] proved the following result.to $\mu^{*}$.

Theorem 1. Let $(X, ð)$ be a complete metric space and $T: X \longrightarrow \mathrm{CB}(X)$ be a multivalued mapping. Suppose that there exist $\theta \in \Xi$ and $k \in(0,1)$ such that

$$
\begin{aligned}
\mu_{1}, \mu_{2} & \in X, H_{d}\left(T\left(\mu_{1}\right), T\left(\mu_{2}\right)\right)>0 \\
& \Rightarrow \theta\left[H_{d}\left(T\left(\mu_{1}\right), T\left(\mu_{2}\right)\right] \leq\left[\theta\left(ð\left(\mu_{1}, \mu_{2}\right)\right)\right]^{k},\right.
\end{aligned}
$$


where $\Xi$ is the set of mapping $\theta:(0, \infty) \longrightarrow(1, \infty)$ satisfying $\left(\theta_{\mathrm{i}}\right)-\left(\theta_{\mathrm{iii}}\right)$ :

(i) $\left(\theta_{\mathrm{i}}\right) \theta$ is nondecreasing and right-continuous.

(ii) $\left(\theta_{\text {ii }}\right)$ For each $\left\{s_{l}\right\}$ in $(0, \infty), \lim _{l} \rightarrow \infty \theta\left(s_{l}\right)=$ $1 \Leftrightarrow \lim _{l \longrightarrow \infty}\left(s_{l}\right)=0$.

(iii) $\left(\theta_{\text {iii }}\right)$ There exist $r \in(0,1)$ and $\mu \in(0,+\infty]$ such that $\lim _{s \longrightarrow 0^{+}} \theta(s)-1 / s^{r}=\mu$. Then, $T$ has at least one fixed point.

Remark 1 . Let $(X, ð)$ be a metric space. If $T: X \longrightarrow \mathrm{CB}(X)$ is a multivalued mapping satisfying the above theorem, then $\ln \theta\left(H_{d}\left(T\left(\mu_{1}\right), T\left(\mu_{2}\right)\right)\right) \leq k \ln \theta\left(ð\left(\mu_{1}, \mu_{2}\right)\right)<\ln \theta\left(ð\left(\mu_{1}, \mu_{2}\right)\right)$.

Since $\theta$ is nondecreasing, we obtain

$$
H_{d}\left(T \mu_{1}, T \mu_{2}\right)<\partial\left(\mu_{1}, \mu_{2}\right), \quad \text { for all } \mu_{1}, \mu_{2} \in X, T \mu_{1} \neq T \mu_{2} \text {. }
$$

Example 1. The functions $\theta_{1}, \theta_{2}:(0, \infty) \longrightarrow(1, \infty)$, defined by $\theta_{1}(r)=e^{\sqrt{r}}$ and $\theta_{2}(r)=1+\sqrt{r}$, are in $\Xi$.

Lemma 1 (see [6]). Let $(X, ð)$ be a metric space and $\beta_{1}, \beta_{2} \in \mathrm{CB}(X)$ with $H_{d}\left(\beta_{1}, \beta_{2}\right)>0$. Then, for all $h>1$ and $\mu \in \beta_{1}$, there exists $\nu=\nu(\mu) \in \beta_{2}$ such that

$$
\text { ð }(\mu, \nu)<h H_{d}\left(\beta_{1}, \beta_{2}\right) \text {. }
$$

Lemma 2 (see [6]). Let (X,ð) be a metric space, $\beta_{2} \in \mathrm{CB}(X)$, and $\mu \in X$. Then, for each $\epsilon>0$, there exists $\nu \in \beta_{2}$ such that

$$
\text { ð }(\mu, v) \leq ð\left(\mu, \beta_{2}\right)+\varepsilon .
$$

Definition 1 (see [2]). Mapping $\Gamma: \mathbb{R}^{+} \times \mathbb{R}^{+} \longrightarrow \mathbb{R}$ is called a simulation function such that

$$
\begin{aligned}
& (\Gamma 1) \Gamma(0,0)=0 . \\
& (\Gamma 2) \Gamma\left(\mu_{1}, \mu_{2}\right)<\mu_{2}-\mu_{1} \text { for all } \mu_{1}, \mu_{2}>0 . \\
& (\Gamma 3) \text { If }\left\{\mu_{1 \iota}\right\},\left\{\mu_{2 \iota}\right\} \in(0, \infty) \text { such that } \lim _{\iota \rightarrow \infty} \mu_{1 \iota}= \\
& \lim _{l \rightarrow \infty} \mu_{2 \iota}>0 \text {, then }
\end{aligned}
$$

$$
\limsup _{\iota \rightarrow \infty} \Gamma\left(\mu_{1 \imath}, \mu_{2 \iota}\right)<0 .
$$

Due to $(\Gamma 2)$, we have $\Gamma\left(\mu_{1}, \mu_{1}\right)<0$ for all $\mu_{1}>0$. Here, the set $\nabla$ satisfies the conditions $(\Gamma 1)-(\Gamma 3)$.

Example 2 (see [2]). For $j=1,2$, let $\vartheta_{j}: \mathbb{R}^{+} \longrightarrow \mathbb{R}^{+}$be continuous functions such that $\vartheta_{j}\left(\mu_{1}\right)=0 \Leftrightarrow \mu_{1}=0$. Functions $\Gamma_{j}: \mathbb{R}^{+} \times \mathbb{R}^{+} \longrightarrow \mathbb{R}(j=1,2)$ are in $\nabla$ :

(i) $\Gamma_{1}\left(\mu_{1}, \mu_{2}\right)=\vartheta_{1}\left(\mu_{2}\right)-\vartheta_{2}\left(\mu_{1}\right)$ for all $\mu_{1}, \mu_{2} \geq 0$, where $\vartheta_{1}\left(\mu_{1}\right) \leq \mu_{1} \leq \vartheta_{2}\left(\mu_{1}\right)$. (ii) $\Gamma_{2}\left(\mu_{1}, \mu_{2}\right)=\mu_{2}-\int_{0}^{\mu_{1}} \varsigma(u) \mathrm{d} u$ for all $\mu_{1}, \mu_{2} \geq 0$, where $\varsigma: \mathbb{R}^{+} \longrightarrow \mathbb{R}^{+}$is a function such that

$\int_{0}^{\varepsilon} \varsigma(u) \mathrm{d} u$ exists and $\int_{0}^{\varepsilon} \varsigma(u) \mathrm{d} u>\varepsilon$ for all $\varepsilon>0$,

Definition 2 (see [1]). Let $(X, ð)$ be a metric space and $\Lambda$ be a nonempty subset of $X$, and $T: \Lambda \longrightarrow \mathrm{CB}(X)$ is known as $\alpha$-admissible, if there exists a mapping $\alpha: \Lambda \times \Lambda \longrightarrow[0, \infty)$ such that

$$
\alpha\left(\beta_{1}, \beta_{2}\right) \geq 1 \Rightarrow \alpha(\mu, \nu) \geq 1,
$$

for all $\mu \in T\left(\beta_{1}\right) \cap \Lambda$ and $\nu \in T\left(\beta_{2}\right) \cap \Lambda$.

Lemma 3 (see [7]). Suppose there is a point $\mu_{0} \in \Lambda$ ( $\Lambda$ is a closed subset of $X)$ that satisfies

$$
\text { ð }\left(\mu_{0}, T\left(\mu_{0}\right)\right) \in \widehat{E},
$$

and $\mu_{\iota} \in \Lambda$ for some $\iota \geq 0$. Then, $ð\left(\mu_{t}, T\left(\mu_{l}\right)\right) \in \widehat{E}$ where $\widehat{E}$ denotes an interval on $\mathbb{R}^{+}$containing 0 .

Definition 3 (see [7]). (inclusion ball) Suppose $\mu_{0} \in \Lambda$ and ð $\left(\mu_{0}, T\left(\mu_{0}\right)\right) \in \widehat{E}$. Then, for all iterates $\mu_{\iota}(\iota \geq 0)$ which belongs to $\Lambda$, we define the closed-ball $\bar{b}\left(\mu_{t}, \rho_{t}\right)$ with center $\mu_{t}$ and radius $\rho_{l}=\sigma\left(ð\left(\mu_{l}, T\left(\mu_{l}\right)\right)\right)$, where $\sigma: \widehat{E} \longrightarrow R_{+}$is defined by (13).

Lemma 4 (see [7]). Suppose there is a point $\mu_{0} \in \Lambda$ that satisfies $\partial\left(\mu_{0}, T\left(\mu_{0}\right)\right) \in \widehat{E}$ and $\bar{b}\left(\mu_{l}, \rho_{l}\right) \subset \Lambda$ for some $\iota \geq 0$; then, $\mu_{t+1} \in \Lambda$ and $\bar{b}\left(\mu_{t+1}, \rho_{t+1}\right) \subset \bar{b}\left(\mu_{l}, \rho_{l}\right)$.

Definition 4 (see [7]). Let $j \geq 1$, and $\eta: \widehat{E} \longrightarrow \widehat{E}$ is known as a gauge function of order $j$ on $\widehat{E}$, if it satisfies the following conditions:

(i) $\eta(\lambda \mu)<\lambda^{j} \eta(\mu)$ for all $\lambda \in(0,1)$ and $\mu \in \widehat{E}$.

(ii) $\eta(\mu)<\mu$ for all $\mu \in \widehat{E}-\{0\}$.

Note that the first condition of Definition 4 is equivalent to $\eta(0)=0$ and $\eta(\mu) / \mu^{i}$ is nondecreasing on $\widehat{E}-\{0\}$.

Definition 5 (see [7]). A gauge function $\eta: \widehat{E} \longrightarrow \widehat{E}$ is said to be a Bianchini-Grandolfi gauge function on $\widehat{E}$ if

$$
\sigma(\mu)=\sum_{i=0}^{\infty} \eta^{i}(\mu)<\infty, \quad \text { for all } \mu \in \widehat{E} .
$$

Note that a Bianchini-Grandolfi gauge function also satisfies the following functional equation:

$$
\sigma(\mu)=\sigma(\eta(\mu))+\mu .
$$

\section{Set-Valued SU $\boldsymbol{\theta}_{\boldsymbol{\eta}}$-Contraction}

The first main definition of this exposition is as follows. 
Definition 6. Let $(X, ð)$ be a metric space, $\Lambda$ be a closed subset of $X$, and $\eta$ be a Bianchini-Grandolfi gauge function on $\widehat{E}$. A mapping $T: \Lambda \longrightarrow \mathrm{CB}(X)$ is known as set-valued SU $\theta_{\eta}$-contraction, if there exists $\theta \in \Xi$ such that for $T(\mu) \cap \Lambda \neq \varnothing$,

$$
\frac{1}{2} \min \{ð(\mu, T(\mu) \cap \Lambda), ð(\nu, T(\nu) \cap \Lambda)\}<ð(\mu, \nu),
$$

which implies that

$$
\theta\left[H_{d}(T(\mu) \cap \Lambda, T(\nu) \cap \Lambda)\right] \leq[\theta(\eta(\Omega(\mu, \nu)))]^{k},
$$

where

$$
\Omega(\mu, \nu)=\max \left\{ð(\mu, \nu), \partial(\mu, T(\mu)), \text { ð }(\nu, T(\nu)), \frac{ð(\mu, T(\nu))+ð(\nu, T(\mu))}{2}\right\},
$$

for all $\mu \in \Lambda, \nu \in T(\mu) \cap \Lambda$ with $ð(\mu, \nu) \in \widehat{E}$, where $k \in(0,1)$.

Theorem 2. Let $(X, ð)$ be a complete me $\theta_{\eta}$ tric space and $T: \Lambda \longrightarrow \mathrm{CB}(X)$ be a multivalued SU -contraction. Suppose $\mu_{0} \in \Lambda$ such that $ð\left(\mu_{0}, c^{*}\right) \in \widehat{E}$ for some $c^{*} \in T\left(\mu_{0}\right) \cap \Lambda$. Then, there exists an orbit $\left\{\mu_{l}\right\}$ of $T$ in $\Lambda$ and $\vartheta^{*} \in \Lambda$ such that $\lim _{\iota \longrightarrow \infty} \mu_{l}=\vartheta^{*}$. In addition, $\vartheta^{*}$ is a fixed point of $T$ if and only if the function $g(\mu):=ð(\mu, T(\mu) \cap \Lambda)$ is T-o.l.s.c at $\vartheta^{*}$.

Proof. Choose $\mu_{1}=c^{*} \in T\left(\mu_{0}\right) \cap \Lambda$. In the case that $ð\left(\mu_{0}, \mu_{1}\right)=0, \mu_{0}$ is a fixed point of $T$. Thus, we assume that $ð\left(\mu_{0}, \mu_{1}\right) \neq 0$. On the other hand, we have

$$
\frac{1}{2} \min \left\{ð\left(\mu_{0}, T\left(\mu_{0}\right) \cap \Lambda\right), \partial\left(\mu_{1}, T\left(\mu_{1}\right) \cap \Lambda\right)\right\}<ð\left(\mu_{0}, \mu_{1}\right) .
$$

Define $\rho=\sigma\left(ð\left(\mu_{0}, \mu_{1}\right)\right)$. From (13), we have $\sigma(r) \geq r$. Hence, $\partial\left(\mu_{0}, \mu_{1}\right) \leq \rho$, and so $\mu_{1} \in \bar{b}\left(\mu_{0}, \rho\right)$. Since $ð\left(\mu_{0}, \mu_{1}\right) \in \widehat{E}$, from (15) and (17), it follows that

$$
\begin{aligned}
\theta\left[H_{d}\left(T\left(\mu_{0}\right) \cap \Lambda, T\left(\mu_{1}\right) \cap \Lambda\right)\right] & \leq\left[\theta\left(\eta\left(ð\left(\mu_{0}, \mu_{1}\right)\right)\right)\right]^{k} \\
& <\left[\theta\left(\Omega\left(\mu_{0}, \mu_{1}\right)\right)\right]^{k} .
\end{aligned}
$$

By right continuity of $\theta$, there exists a real number $h_{1}>1$ such that

$$
\theta\left[h_{1} H_{d}\left(T\left(\mu_{0}\right) \cap \Lambda, T\left(\mu_{1}\right) \cap \Lambda\right)\right] \leq\left[\theta\left(\Omega\left(\mu_{0}, \mu_{1}\right)\right)\right]^{k} .
$$

From

$$
\begin{aligned}
\text { ð }\left(\mu_{1}, T\left(\mu_{1}\right) \cap \Lambda\right) & \leq H_{d}\left(T\left(\mu_{0}\right) \cap \Lambda, T\left(\mu_{1}\right) \cap \Lambda\right) \\
& <h_{1} H_{d}\left(T\left(\mu_{0}\right) \cap \Lambda, T\left(\mu_{1}\right) \cap \Lambda\right),
\end{aligned}
$$

by Lemma 1 , there exists $\mu_{2} \in T\left(\mu_{1}\right) \cap \Lambda$ such that $ð\left(\mu_{1}, \mu_{2}\right) \leq h_{1} H_{d}\left(T\left(\mu_{0}\right) \cap \Lambda, T\left(\mu_{1}\right) \cap \Lambda\right)$. Since $\theta$ is nondecreasing, by (19), this inequality gives that

$$
\theta\left(ð\left(\mu_{1}, \mu_{2}\right)\right) \leq \theta\left[h_{1} H_{d}\left(T\left(\mu_{0}\right) \cap \Lambda, T\left(\mu_{1}\right) \cap \Lambda\right)\right] \leq\left[\theta\left(\Omega\left(\mu_{0}, \mu_{1}\right)\right)\right]^{k},
$$

where

$$
\begin{aligned}
\Omega\left(\mu_{0}, \mu_{1}\right) & =\max \left\{ð\left(\mu_{0}, \mu_{1}\right), ð\left(\mu_{0}, T\left(\mu_{0}\right)\right), ð\left(\mu_{1}, T\left(\mu_{1}\right)\right), \frac{ð\left(\mu_{0}, T\left(\mu_{1}\right)\right)+ð\left(\mu_{1}, T\left(\mu_{0}\right)\right)}{2}\right\} \\
& \leq \max \left\{ð\left(\mu_{0}, \mu_{1}\right), ð\left(\mu_{1}, T\left(\mu_{1}\right)\right), \frac{\partial\left(\mu_{0}, T\left(\mu_{1}\right)\right)}{2}\right\} \\
& \leq \max \left\{ð\left(\mu_{0}, \mu_{1}\right), ð\left(\mu_{1}, T\left(\mu_{1}\right)\right)\right\} .
\end{aligned}
$$

We claim that

$$
\begin{aligned}
\theta\left(\partial\left(\mu_{1}, \mu_{2}\right)\right) & \leq \theta\left[h_{1} H_{d}\left(T\left(\mu_{0}\right) \cap \Lambda, T\left(\mu_{1}\right) \cap \Lambda\right)\right] \\
& \leq\left[\theta\left(ð\left(\mu_{0}, \mu_{1}\right)\right)\right]^{k} .
\end{aligned}
$$

Let $\Phi=\max \left\{ð\left(\mu_{0}, \mu_{1}\right), ð\left(\mu_{1}, T\left(\mu_{1}\right)\right)\right\}$. If $\Phi=ð\left(\mu_{1}\right.$, $\left.T\left(\mu_{1}\right)\right)$, we have $\mu_{2} \in T\left(\mu_{1}\right) \cap \Lambda$, so we obtain

$$
\theta\left(ð\left(\mu_{1}, \mu_{2}\right)\right) \leq \theta\left[h_{1} H_{d}\left(T\left(\mu_{0}\right) \cap \Lambda, T\left(\mu_{1}\right) \cap \Lambda\right)\right] \leq\left[\theta\left(ð\left(\mu_{1}, \mu_{2}\right)\right)\right]^{k},
$$

which is a contradiction. Thus, we conclude that $\Phi=ð\left(\mu_{0}, \mu_{1}\right)$. We assume that $ð\left(\mu_{1}, \mu_{2}\right) \neq 0$; otherwise, $\mu_{1}$ is a fixed point of $T$. From Remark 1, we have $ð\left(\mu_{1}, \mu_{2}\right)<ð\left(\mu_{0}, \mu_{1}\right)$, and so $\quad\left(\mu_{1}, \mu_{2}\right) \in \widehat{E}$. Next, $\mu_{2} \in \vec{b}\left(\mu_{0}, \rho\right)$ because

$$
\begin{aligned}
ð\left(\mu_{0}, \mu_{2}\right) & \leq ð\left(\mu_{0}, \mu_{1}\right)+ð\left(\mu_{1}, \mu_{2}\right) \\
& \leq ð\left(\mu_{0}, \mu_{1}\right)+\eta\left(ð\left(\mu_{0}, \mu_{1}\right)\right) \\
& \leq ð\left(\mu_{0}, \mu_{1}\right)+\sigma\left(\eta\left(ð\left(\mu_{0}, \mu_{1}\right)\right)\right) \\
& =\sigma\left(ð\left(\mu_{0}, \mu_{1}\right)\right)=\rho .
\end{aligned}
$$




$$
\begin{aligned}
& \text { Also, since } \\
& \frac{1}{2} \min \left\{\partial\left(\mu_{1}, T\left(\mu_{1}\right) \cap \Lambda\right), ð\left(\mu_{2}, T\left(\mu_{2}\right) \cap \Lambda\right)\right\}<ð\left(\mu_{1}, \mu_{2}\right),
\end{aligned}
$$

from (15), we get

$$
\begin{aligned}
\theta\left[H_{d}\left(T\left(\mu_{1}\right) \cap \Lambda, T\left(\mu_{2}\right) \cap \Lambda\right)\right] & \leq\left[\theta\left(\eta\left(ð\left(\mu_{1}, \mu_{2}\right)\right)\right)\right]^{k} \\
& <\left[\theta\left(\Omega\left(\mu_{1}, \mu_{2}\right)\right)\right]^{k} .
\end{aligned}
$$

Since $\theta$ is right-continuous, there exists a real number $h_{2}>1$ such that

$$
\theta\left[h_{2} H_{d}\left(T\left(\mu_{1}\right) \cap \Lambda, T\left(\mu_{2}\right) \cap \Lambda\right)\right] \leq\left[\theta\left(\Omega\left(\mu_{1}, \mu_{2}\right)\right)\right]^{k} .
$$

Next, from

$$
\begin{aligned}
\partial\left(\mu_{2}, T\left(\mu_{2}\right) \cap \Lambda\right) & \leq H_{d}\left(T\left(\mu_{1}\right) \cap \Lambda, T\left(\mu_{2}\right) \cap \Lambda\right) \\
& <h_{2} H_{d}\left(T\left(\mu_{1}\right) \cap \Lambda, T\left(\mu_{2}\right) \cap \Lambda\right),
\end{aligned}
$$

by Lemma 1 , there exists $\mu_{3} \in T\left(\mu_{2}\right) \cap \Lambda$ such that $ð\left(\mu_{2}, \mu_{3}\right) \leq h_{2} H_{d}\left(T\left(\mu_{1}\right) \cap \Lambda, T\left(\mu_{2}\right) \cap \Lambda\right)$. By (28), this inequality gives that

$$
\begin{aligned}
\theta\left(ð\left(\mu_{2}, \mu_{3}\right)\right) & \leq \theta\left[h_{2} H_{d}\left(T\left(\mu_{1}\right) \cap \Lambda, T\left(\mu_{2}\right) \cap \Lambda\right)\right] \\
& \leq\left[\theta\left(\Omega\left(\mu_{1}, \mu_{2}\right)\right)\right]^{k} \leq\left[\theta\left(\Omega\left(\mu_{0}, \mu_{1}\right)\right)\right]^{k^{2}} .
\end{aligned}
$$

where

$$
\begin{aligned}
\Omega\left(\mu_{1}, \mu_{2}\right) & =\max \left\{\partial\left(\mu_{1}, \mu_{2}\right), ð\left(\mu_{1}, T\left(\mu_{1}\right)\right), ð\left(\mu_{2}, T\left(\mu_{2}\right)\right), \frac{\partial\left(\mu_{1}, T\left(\mu_{2}\right)\right)+ð\left(\mu_{2}, T\left(\mu_{1}\right)\right)}{2}\right\} \\
& \leq \max \left\{ð\left(\mu_{1}, \mu_{2}\right), ð\left(\mu_{2}, T\left(\mu_{2}\right)\right), \frac{\partial\left(\mu_{1}, T\left(\mu_{2}\right)\right)}{2}\right\} \\
& \leq \max \left\{ð\left(\mu_{1}, \mu_{2}\right), ð\left(\mu_{2}, T\left(\mu_{2}\right)\right)\right\} .
\end{aligned}
$$

We claim that

$$
\begin{aligned}
\theta\left(ð\left(\mu_{2}, \mu_{3}\right)\right) & \leq \theta\left[h_{2} H_{d}\left(T\left(\mu_{1}\right) \cap \Lambda, T\left(\mu_{2}\right) \cap \Lambda\right)\right] \\
& \leq\left[\theta\left(ð\left(\mu_{1}, \mu_{2}\right)\right)\right]^{k} \leq\left[\theta\left(ð\left(\mu_{0}, \mu_{1}\right)\right)\right]^{k^{2}}
\end{aligned}
$$

Let $\Phi=\max \left\{\partial\left(\mu_{1}, \mu_{2}\right), \partial\left(\mu_{2}, T\left(\mu_{2}\right)\right)\right\}$. $\Phi=ð\left(\mu_{2}, T\left(\mu_{2}\right)\right)$, we have $\mu_{3} \in T\left(\mu_{2}\right) \cap \Lambda$, so we obtain

$$
\theta\left(\text { ð }\left(\mu_{2}, \mu_{3}\right)\right) \leq \theta\left[h_{2} H_{d}\left(T\left(\mu_{1}\right) \cap \Lambda, T\left(\mu_{2}\right) \cap \Lambda\right)\right] \leq\left[\theta\left(ð\left(\mu_{2}, \mu_{3}\right)\right)\right]^{k},
$$

which is a contradiction. Thus, we conclude that $\Phi=ð\left(\mu_{1}, \mu_{2}\right)$. We assume that $ð\left(\mu_{2}, \mu_{3}\right) \neq 0$; otherwise, $\mu_{2}$ is a fixed point of $T$. From Remark 1, we have $ð\left(\mu_{2}, \mu_{3}\right)<ð\left(\mu_{1}, \mu_{2}\right)$, and so $ð\left(\mu_{2}, \mu_{3}\right) \in \widehat{E}$. Also, we have $\mu_{3} \in \vec{b}\left(\mu_{0}, \rho\right)$, since

$$
\begin{aligned}
ð\left(\mu_{0}, \mu_{3}\right) & \leq ð\left(\mu_{0}, \mu_{1}\right)+ð\left(\mu_{1}, \mu_{2}\right)+ð\left(\mu_{2}, \mu_{3}\right) \\
& \leq ð\left(\mu_{0}, \mu_{1}\right)+\eta\left(ð\left(\mu_{0}, \mu_{1}\right)\right)+\eta^{2}\left(ð\left(\mu_{0}, \mu_{1}\right)\right) \\
& \leq \sum_{i=0}^{\infty} \eta^{i}\left(ð\left(\mu_{0}, \mu_{1}\right)\right) \\
& =\sigma\left(ð\left(\mu_{0}, \mu_{1}\right)\right)=\rho .
\end{aligned}
$$

Continuing this setup, we have two sequences $\left\{\mu_{t}\right\} \subset \bar{b}\left(\mu_{0}, \rho\right)$ and $\left\{h_{t}\right\} \subset(1, \infty)$ such that $\mu_{t+1} \in T\left(\mu_{t}\right) \cap \Lambda$, $\mu_{t} \neq \mu_{t+1}$ with $\partial\left(\mu_{t}, \mu_{t+1}\right) \in \widehat{E}$ and

$$
\begin{aligned}
1<\theta\left(\partial\left(\mu_{t}, \mu_{t+1}\right)\right) & \leq \theta\left(h_{t} H_{d}\left(T\left(\mu_{t-1}\right) \cap \Lambda, T\left(\mu_{t}\right) \cap \Lambda\right)\right) \\
& \leq\left[\theta\left(ð\left(\mu_{t-1}, \mu_{t}\right)\right)\right]^{k},
\end{aligned}
$$

for all $\iota \in \mathbb{N}$. Then,

$$
1<\theta\left(ð\left(\mu_{t}, \mu_{t+1}\right)\right) \leq\left[\theta\left(ð\left(\mu_{0}, \mu_{1}\right)\right)\right]^{k^{t}}, \quad \text { for all } \iota \in \mathbb{N},
$$

which gives that

$$
\lim _{l \longrightarrow \infty} \theta\left(ð\left(\mu_{t}, \mu_{l+1}\right)\right)=1,
$$

and by $\left(\theta_{\mathrm{ii}}\right)$, we have

$$
\lim _{\iota \longrightarrow \infty} \partial\left(\mu_{\iota}, \mu_{l+1}\right)=0 .
$$

Next, we prove that $\left\{\mu_{l}\right\}$ is a Cauchy sequence in $X$. Setting $\delta_{l}:=\partial\left(\mu_{t}, \mu_{t+1}\right)$, from $\left(\theta_{i i i}\right)$, there exist $r \in(0,1)$ and $\mu \in(0, \infty]$ such that

$$
\lim _{l \longrightarrow \infty} \frac{\theta\left(\delta_{l}\right)-1}{\left(\delta_{l}\right)^{r}}=\mu .
$$

Take $\lambda \in(0, \mu)$. From the definition of limit, there exists $\iota_{0} \in \mathbb{N}$ such that

$$
\left[\delta_{\iota}\right]^{r} \leq \lambda^{-1}\left[\theta\left(\delta_{\iota}\right)-1\right], \quad \text { for all } \iota>\iota_{0} .
$$

Using (36) and the above inequality,

$$
\iota\left[\delta_{\iota}\right]^{r} \leq \lambda^{-1} \iota\left(\left[\theta\left(\delta_{0}\right)\right]^{k^{\prime}}-1\right), \quad \text { for all } \iota>\iota_{0} .
$$


This implies that

$$
\lim _{l \longrightarrow \infty} \iota\left[\delta_{l}\right]^{r}=\lim _{l \longrightarrow \infty} \iota\left[d\left(\mu_{l}, \mu_{l+1}\right)\right]^{r}=0 .
$$

Hence, there exists $\iota_{1} \in \mathbb{N}$ such that

$$
d\left(\mu_{\iota}, \mu_{t+1}\right) \leq \frac{1}{l^{1 / r}}, \quad \text { for all } \iota>\iota_{1} .
$$

Let $p>\iota>\iota_{1}$. Then, using the triangular inequality and (43), we get

$$
ð\left(\mu_{\iota}, \mu_{p}\right) \leq \sum_{j=\iota}^{p-1} \partial\left(\mu_{j}, \mu_{j+1}\right) \leq \sum_{j=\iota}^{p-1} \frac{1}{j^{1 / r}}<\sum_{j=\iota}^{\infty} \frac{1}{j^{1 / r}} .
$$

Owing to the convergence of the series $\sum_{j=l}^{\infty} 1 / j^{1 / r},\left\{\mu_{l}\right\}$ is a Cauchy sequence in $\bar{b}\left(\mu_{0}, \rho\right)$. Since $\bar{b}\left(\mu_{0}, \rho\right)$ is closed in $X$, there exists $\vartheta^{*} \in \bar{b}\left(\mu_{0}, \rho\right)$ such that $\mu_{l} \longrightarrow \vartheta^{*}$. Note that $\vartheta^{*} \in \Lambda$ because $\mu_{l+1} \in T\left(\mu_{l}\right) \cap \Lambda$. Now, we claim that

$$
\frac{1}{2} \min \left\{\partial\left(\mu_{\iota}, T\left(\mu_{\iota}\right) \cap \Lambda\right), \partial\left(\vartheta^{*}, T\left(\vartheta^{*}\right) \cap \Lambda\right)\right\}<\partial\left(\mu_{\iota}, \vartheta^{*}\right),
$$

$$
\begin{aligned}
& \text { or } \\
& \frac{1}{2} \min \left\{ð\left(\vartheta^{*}, T\left(\vartheta^{*}\right) \cap \Lambda\right), ð\left(\mu_{t+1}, T\left(\mu_{t+1}\right) \cap \Lambda\right)\right\}<ð\left(\mu_{t+1}, \vartheta^{*}\right),
\end{aligned}
$$

for all $\iota \in N$. Assume, on the contrary, that there exists $\iota^{\prime} \in N$ such that

$$
\frac{1}{2} \min \left\{ð\left(\mu_{\iota^{\prime}}, T\left(\mu_{\iota^{\prime}}\right) \cap \Lambda\right), \text { ठ }\left(\vartheta^{*}, T\left(\vartheta^{*}\right) \cap \Lambda\right)\right\} \geq ð\left(\mu_{\iota^{\prime}}, \vartheta^{*}\right),
$$

and

$\frac{1}{2} \min \left\{ð\left(\vartheta^{*}, T\left(\vartheta^{*}\right) \cap \Lambda\right)\right.$, ð $\left.\left(\mu_{\iota^{\prime}+1}, T\left(\mu_{\iota^{\prime}+1}\right) \cap \Lambda\right)\right\} \geq ð\left(\mu_{\iota^{\prime}+1}, \vartheta^{*}\right)$.

By (47), we have

$$
\begin{aligned}
& 2 ð\left(\mu_{\iota^{\prime}}, \vartheta^{*}\right) \leq \min \left\{ð\left(\mu_{\iota^{\prime}}, T\left(\mu_{\prime^{\prime}}\right) \cap \Lambda\right), \text { ð }\left(\vartheta^{*}, T\left(\vartheta^{*}\right) \cap \Lambda\right)\right\} \\
& \leq \min \left\{\left[ð\left(\mu_{\iota^{\prime}}, \vartheta^{*}\right)+ð\left(\vartheta^{*}, T\left(\mu_{\iota^{\prime}}\right) \cap \Lambda\right)\right],\right. \\
& \text { ð } \left.\left(\vartheta^{*}, T\left(\vartheta^{*}\right) \cap \Lambda\right)\right\} \\
& \leq\left[ð\left(\mu_{\prime}, \vartheta^{*}\right)+ð\left(\vartheta^{*}, T\left(\mu_{\prime}\right) \cap \Lambda\right)\right] \\
& <\left[ð\left(\mu_{\iota^{\prime}}, \vartheta^{*}\right)+\text { ð }\left(\vartheta^{*}, T\left(\mu_{\iota^{\prime}}\right)\right)\right] \\
& \leq\left[ð\left(\mu_{\iota^{\prime}}, \vartheta^{*}\right)+ð\left(\vartheta^{*}, \mu_{\iota^{\prime}+1}\right)\right] \text {, }
\end{aligned}
$$

which implies that

$$
\text { ð }\left(\mu_{\iota^{\prime}}, \vartheta^{*}\right) \leq ð\left(\vartheta^{*}, \mu_{\iota^{\prime}+1}\right),
$$

which together with (38) gives

$$
\begin{aligned}
\text { ð }\left(\mu_{\iota^{\prime}}, \vartheta^{*}\right) & \leq ð\left(\vartheta^{*}, \mu_{\iota^{\prime}+1}\right) \\
& \leq \frac{1}{2} \min \left\{ð\left(\vartheta^{*}, T\left(\vartheta^{*}\right) \cap \Lambda\right), ð\left(\mu_{\iota^{\prime}+1}, T\left(\mu_{\iota^{\prime}+1}\right) \cap \Lambda\right)\right\} .
\end{aligned}
$$

Since

$$
\frac{1}{2} \min \left\{ð\left(\mu_{\iota^{\prime}}, T\left(\mu_{\iota^{\prime}}\right) \cap \Lambda\right), \text { ठ }\left(\mu_{\iota^{\prime}+1}, T\left(\mu_{\imath^{\prime}+1}\right) \cap \Lambda\right)\right\}<ð\left(\mu_{\iota^{\prime}}, \mu_{\iota^{\prime}+1}\right),
$$

from contractive condition (15), we have

$$
\begin{aligned}
\theta\left(ð\left(\mu_{\iota^{\prime}+1}, \mu_{\iota^{\prime}+2}\right)\right) & \leq \theta\left[h_{2} H_{d}\left(T\left(\mu_{\iota^{\prime}}\right) \cap \Lambda, T\left(\mu_{\iota^{\prime}+1}\right) \cap \Lambda\right)\right] \\
& \leq\left[\theta\left(\Omega\left(\mu_{\iota^{\prime}}, \mu_{\iota^{\prime}+1}\right)\right)\right]^{k}
\end{aligned}
$$

where

$$
\begin{aligned}
\Omega\left(\mu_{i^{\prime}}, \mu_{i^{\prime}+1}\right) & =\max \left\{\begin{array}{c}
\partial\left(\mu_{i^{\prime}}, \mu_{i^{\prime}+1}\right), \partial\left(\mu_{i^{\prime}}, T\left(\mu_{i^{\prime}}\right)\right), \partial\left(\mu_{i^{\prime}+1}, T\left(\mu_{i^{\prime}+1}\right)\right), \\
\frac{\partial\left(\mu_{i^{\prime}}, T\left(\mu_{i^{\prime}+1}\right)\right)+\partial\left(\mu_{i^{\prime}+1}, T\left(\mu_{i^{\prime}}\right)\right)}{2}
\end{array}\right\} \\
& \leq \max \left\{\begin{array}{c}
ð\left(\mu_{i^{\prime}}, \mu_{i^{\prime}+1}\right), \partial\left(\mu_{i^{\prime}+1}, \mu_{i^{\prime}+2}\right), \\
\frac{\partial\left(\mu_{i^{\prime}}, \mu_{i^{\prime}+2}\right)}{2}
\end{array}\right\} \\
& \leq \max \left\{ð\left(\mu_{i^{\prime}}, \mu_{i^{\prime}+1}\right), \partial\left(\mu_{i^{\prime}+1}, \mu_{i^{\prime}+2}\right)\right\} .
\end{aligned}
$$

We claim that

$$
\begin{aligned}
\theta\left(ð\left(\mu_{\iota^{\prime}+1}, \mu_{\iota^{\prime}+2}\right)\right) & \leq \theta\left[h_{2} H_{d}\left(T\left(\mu_{\iota^{\prime}}\right) \cap \Lambda, T\left(\mu_{\iota^{\prime}+1}\right) \cap \Lambda\right)\right] \\
& \leq\left[\theta\left(ð\left(\mu_{\iota^{\prime}}, \mu_{\iota^{\prime}+1}\right)\right)\right]^{k} .
\end{aligned}
$$

Let $\Delta=\max \left\{ð\left(\mu_{\iota^{\prime}}, \mu_{l^{\prime}+1}\right), ð\left(\mu_{l^{\prime}+1}, \mu_{\iota^{\prime}+2}\right)\right\}$. If $\Delta=ð\left(\mu_{l^{\prime}+1}\right.$, $\left.\mu_{i^{\prime}+2}\right)$. Since $\mu_{l^{\prime}+2} \in T\left(\mu_{\imath^{\prime}+1}\right) \cap \Lambda$, we have

$$
\begin{aligned}
\theta\left(ð\left(\mu_{\iota^{\prime}+1}, \mu_{\iota^{\prime}+2}\right)\right) & \leq \theta\left[h_{2} H_{d}\left(T\left(\mu_{\iota^{\prime}}\right) \cap \Lambda, T\left(\mu_{\iota^{\prime}+1}\right) \cap \Lambda\right)\right] \\
& \leq\left[\theta\left(ð\left(\mu_{\iota^{\prime}+1}, \mu_{\iota^{\prime}+2}\right)\right)\right]^{k},
\end{aligned}
$$

which is a contradiction. Thus, we conclude that $\Delta=ð\left(\mu_{\iota^{\prime}}, \mu_{\iota^{\prime}+1}\right)$. From Remark 1, we have

$$
\text { ð }\left(\mu_{i^{\prime}+1}, \mu_{\iota^{\prime}+2}\right)<ð\left(\mu_{\iota^{\prime}}, \mu_{i^{\prime}+1}\right) .
$$

From (38), (43), and (47), we obtain

$$
\begin{aligned}
ð\left(\mu_{i^{\prime}+1}, \mu_{i^{\prime}+2}\right) & <\text { ठ }\left(\mu_{i^{\prime}}, \mu_{i^{\prime}+1}\right) \\
& \leq\left[ð\left(\mu_{i^{\prime}}, \vartheta^{*}\right)+ð\left(\vartheta^{*}, \mu_{i^{\prime}+1}\right)\right] \\
& \leq\left[\begin{array}{l}
\frac{1}{2} \min \left\{ð\left(\vartheta^{*}, T\left(\vartheta^{*}\right) \cap \Lambda\right), ð\left(\mu_{i^{\prime}+1}, T\left(\mu_{i^{\prime}+1}\right) \cap \Lambda\right)\right\} \\
1 \\
+\frac{1}{2} \min \left\{ð\left(\vartheta^{*}, T\left(\vartheta^{*}\right) \cap \Lambda\right), ð\left(\mu_{i^{\prime}+1}, T\left(\mu_{i^{\prime}+1}\right) \cap \Lambda\right)\right\}
\end{array}\right] \\
& \leq \min \left\{ð\left(\vartheta^{*}, T\left(\vartheta^{*}\right) \cap \Lambda\right), ð\left(\mu_{i^{\prime}+1}, T\left(\mu_{i^{\prime}+1}\right) \cap \Lambda\right)\right\} \\
& \leq ð\left(\mu_{i^{\prime}+1}, T\left(\mu_{i^{\prime}+1}\right) \cap \Lambda\right),
\end{aligned}
$$

which is a contradiction. Hence, (45) holds true: 


$$
\frac{1}{2} \min \left\{ð\left(\mu_{l}, T\left(\mu_{\iota}\right) \cap \Lambda\right), ð\left(\vartheta^{*}, T\left(\vartheta^{*}\right) \cap \Lambda\right)\right\}<ð\left(\mu_{l}, \vartheta^{*}\right) \quad \text { for all } \iota \geq 2 \text {. }
$$

Also, we know that $ð\left(\mu_{t}, \mu_{t+1}\right) \in \widehat{E}$ for all $n$. Thus, from where (15), we have

$$
\begin{aligned}
\theta\left(ð\left(\mu_{l+1}, T\left(\mu_{l+1}\right) \cap \Lambda\right)\right) & \leq \theta\left[H_{d}\left(T\left(\mu_{\imath}\right) \cap \Lambda, T\left(\mu_{l+1}\right) \cap \Lambda\right)\right] \\
& \leq\left[\theta\left(\eta\left(\Omega\left(\mu_{\imath}, \mu_{l+1}\right)\right)\right)\right]^{k} \\
& <\theta\left[\left(\Omega\left(\mu_{l}, \mu_{l+1}\right)\right)\right]^{k},
\end{aligned}
$$

$$
\begin{aligned}
\Omega\left(\mu_{t}, \mu_{t+1}\right) & =\max \left\{\begin{array}{c}
\partial\left(\mu_{t}, \mu_{t+1}\right), \partial\left(\mu_{t}, T\left(\mu_{t}\right)\right), \partial\left(\mu_{t+1}, T\left(\mu_{t+1}\right)\right), \\
\frac{\partial\left(\mu_{t}, T\left(\mu_{t+1}\right)\right)+\partial\left(\mu_{t+1}, T\left(\mu_{t}\right)\right)}{2}
\end{array}\right\} \\
& \leq \max \left\{\begin{array}{c}
ð\left(\mu_{t}, \mu_{t+1}\right), ð\left(\mu_{t+1}, \mu_{t+2}\right), \\
\frac{\partial\left(\mu_{t}, \mu_{t+2}\right)}{2}
\end{array}\right\} \\
& \leq \max \left\{\partial\left(\mu_{t}, \mu_{t+1}\right), \partial\left(\mu_{t+1}, \mu_{t+2}\right)\right\} .
\end{aligned}
$$

We claim that

$$
\begin{aligned}
\theta\left(ð\left(\mu_{t+1}, T\left(\mu_{\iota+1}\right) \cap \Lambda\right)\right) & \leq \theta\left[H_{d}\left(T\left(\mu_{\iota}\right) \cap \Lambda, T\left(\mu_{\iota+1}\right) \cap \Lambda\right)\right] \\
& <\theta\left[\left(ð\left(\mu_{\iota}, \mu_{\iota+1}\right)\right)\right]^{k} .
\end{aligned}
$$

Let $\Phi=\max \left\{ð\left(\mu_{l}, \mu_{t+1}\right), ð\left(\mu_{t+1}, \mu_{t+2}\right)\right\}$ If $\Phi=ð\left(\mu_{t+1}, \mu_{t+2}\right)$, we have $\mu_{t+2} \in T\left(\mu_{t+1}\right) \cap \Lambda$, so we obtain

$$
\begin{aligned}
\theta\left(ð\left(\mu_{t+1}, T\left(\mu_{t+1}\right) \cap \Lambda\right)\right) & \leq \theta\left[H_{d}\left(T\left(\mu_{\iota}\right) \cap \Lambda, T\left(\mu_{t+1}\right) \cap \Lambda\right)\right] \\
& <\theta\left[\left(ð\left(\mu_{t+1}, \mu_{t+2}\right)\right)\right]^{k},
\end{aligned}
$$

which is a contradiction. Thus, we obtain $\Phi=ð\left(\mu_{l}, \mu_{t+1}\right)$. From Remark 1, we deduce

$$
\text { ð }\left(\mu_{t+1}, T\left(\mu_{t+1}\right) \cap \Lambda\right)<ð\left(\mu_{l}, \mu_{t+1}\right) .
$$

Taking limit $\iota \longrightarrow \infty$ in (64),

$$
\lim _{l \longrightarrow \infty} ð\left(\mu_{l+1}, T\left(\mu_{t+1}\right) \cap \Lambda\right)=0 .
$$

Since $g(\mu)=ð(\mu, T(\mu) \cap \Lambda)$ is $T$-o.l.s.c at $\vartheta^{*}$, then

$$
\begin{aligned}
\text { ठ }\left(\vartheta^{*}, T\left(\vartheta^{*}\right) \cap \Lambda\right) & =g\left(\vartheta^{*}\right) \leq \liminf _{\iota} g\left(\mu_{l+1}\right) \\
& =\liminf _{\iota} \partial\left(\mu_{l+1}, T\left(\mu_{l+1}\right) \cap \Lambda\right)=0 .
\end{aligned}
$$

Since $T\left(\vartheta^{*}\right)$ is closed, we have $\vartheta^{*} \in T\left(\vartheta^{*}\right)$. Conversely, if $\vartheta^{*}$ is a fixed point of $T$, then $g\left(\vartheta^{*}\right)=0 \leq \liminf _{l} g\left(\mu_{l}\right)$, since $\vartheta^{*} \in \Lambda$.

Corollary 1. Let $(X, ð)$ be a complete metric space, $\eta$ be a Bianchini-Grandolfi gauge function on an interval $\widehat{E}$, and $T: \Lambda \longrightarrow C B(X)$ be a given set-valued mapping. If $k \in(0,1)$ and $T(\mu) \cap \Lambda \neq \varnothing$ exist,

$$
\frac{1}{2} \min \{ð(\mu, T(\mu) \cap \Lambda), ð(\nu, T(\nu) \cap \Lambda)\}<ð(\mu, \nu),
$$

which implies that

$$
\theta\left[H_{d}(T(\mu) \cap \Lambda, T \nu \cap \Lambda)\right] \leq[\theta(\eta(ð(\mu, \nu)))]^{k},
$$

for all $\mu \in \Lambda, \nu \in T(\mu) \cap \Lambda$ with $ð(\mu, \nu) \in \widehat{E}$. Suppose $\mu_{0} \in \Lambda$ such that $\partial\left(\mu_{0}, c^{*}\right) \in \widehat{E}$ for some $c^{*} \in T\left(\mu_{0}\right) \cap \Lambda$. Then, there exists an orbit $\left\{\mu_{l}\right\}$ of $T$ in $\Lambda$ and $\vartheta^{*} \in \Lambda$ such that $\lim _{\iota \longrightarrow \infty} \mu_{l}=9^{*}$. In addition, $\vartheta^{*}$ is a fixed point of $T$ if and only if the function $g(\mu):=ð(\mu, T(\mu) \cap \Lambda)$ is T-o.l.s.c at point $\vartheta^{*}$.

Corollary 2. Let $(X, ð)$ be a complete metric space, $\eta$ be a Bianchini-Grandolfi gauge function on an interval $\widehat{E}$, and $T: \Lambda \longrightarrow C B(X)$ be a given set-valued mapping. If $k \in(0,1)$ and for $T \mu \cap \Lambda \neq \varnothing$ exist, 


$$
\frac{1}{2} \min \{ð(\mu, T(\mu) \cap \Lambda), ð(\nu, T(\nu) \cap \Lambda)\}<ð(\mu, \nu),
$$

implies that

$$
\sqrt{H_{d}(T(\mu) \cap \Lambda, T(\nu) \cap \Lambda)} \leq k \sqrt{\eta(\partial(\mu, \nu))},
$$

for all $\mu \in \Lambda, \nu \in T(\mu) \cap \Lambda$, and $\partial(\mu, \nu) \in \widehat{E}$. In addition, suppose $\mu_{0} \in \Lambda$ such that $ð\left(\mu_{0}, c^{*}\right) \in \widehat{E}$ for some $c^{*} \in T\left(\mu_{0}\right) \cap \Lambda$. Then, there exists an orbit $\left\{\mu_{t}\right\}$ of $T$ in $\Lambda$, $\vartheta^{*} \in \Lambda$ such that $\lim _{l \rightarrow \infty} \mu_{l}=\vartheta^{*}$ and $\vartheta^{*}$ is a fixed point of $T$ if and only if the function $g(\mu):=ð(\mu, T(\mu) \cap \Lambda)$ is T-o.l.s.c at $\vartheta^{*}$.

Corollary 3. Let $(X, ð)$ be a complete metric space, $\eta$ be a Bianchini-Grandolfi gauge function on $\widehat{E}$, and $T: \Lambda \longrightarrow C B(X)$ be a given set-valued mapping. If $\theta \in \Xi$ and $k \in(0,1)$ exist, then

$$
\begin{array}{r}
\frac{1}{2} \min \{\partial(\mu, T(\mu) \cap \Lambda), ð(\nu, T(\nu) \cap \Lambda)\}<ð(\mu, \nu), \\
\Rightarrow \theta\left[H_{d}(T(\mu), T(\nu))\right] \leq[\theta(\eta(ð(\mu, \nu)))]^{k},
\end{array}
$$

for all $\mu \in X, \nu \in T(\mu)$, and $ð(\mu, \nu) \in \widehat{E}$. Suppose that $\mu_{0} \in X$ such that $ð\left(\mu_{0}, c^{*}\right) \in \widehat{E}$ for some $c^{*} \in T \mu_{0}$. Then, there exists an orbit $\left\{\mu_{l}\right\}$ of $T$ in $X$ that converges to the fixed point $\vartheta^{*} \in \mathscr{F}=\left\{\mu \in X: ð\left(\mu, \vartheta^{*}\right) \in \widehat{E}\right\}$ of $T$.

Example 3. Let $X=[-10, \infty)$ be an usual metric $ð$ and let $\widehat{E}=[0, \infty)$. Mapping $T: \Lambda \longrightarrow \mathrm{CB}(X)$ is defined as

$$
T(\mu)= \begin{cases}{\left[0, \frac{\mu}{8}\right],} & \mu \in[0,4], \\ \{0, \mu\}, & \mu \in[-10,0) \cup(4, \infty) .\end{cases}
$$

Clearly, $\quad 1 / 2 \min \{ð(\mu, T(\mu) \cap \Lambda), ð(\nu, T(\nu) \cap \Lambda)\}<$ $ð(\mu, \nu)$ if and only if $\mu, \nu \in[0,4]$. Let $\mu_{0}=4$; then, we have $c^{*}=1 / 2 \in T\left(\mu_{0}\right)$ such that $ठ\left(\mu_{0}, c^{*}\right) \in \widehat{E}$. Firstly, we claim that $T$ satisfies inequality (68) with setting $\theta(r)=e^{\sqrt{r e^{r}}}$, $\eta(r)=r / 2$, and $k=1 / 2$. For $\mu \in[0,4]$ and $\nu \in T(\mu)$, we obtain

$$
\begin{aligned}
\theta\left[H_{d}(T(\mu), T(\nu))\right] & =\theta\left(\frac{|\mu-\nu|}{8}\right) \\
& =e^{\sqrt{|\mu-\nu| / 8 e^{|\mu-\nu| / 8}}} \\
& \leq e^{1 / 2 \sqrt{|\mu-\nu| / 2 e^{|\mu-\nu| / 2}}} \\
& =e^{1 / 2 \sqrt{\eta(\widehat{d}(v, v)) e^{\eta(\hat{d}(v, v))}}} \\
& =[\theta(\eta(ð(\mu, \nu)))]^{k} .
\end{aligned}
$$

Consequently, the requirements of Corollary 1 are fulfilled and 0 is a fixed point of $T$. For $\mu=0$ and $\nu=5$,

$$
\begin{aligned}
\theta\left[H_{d}(T(\mu), T(\nu))\right] & =\theta\left[H_{d}(T(0), T(5))\right] \\
& =\theta(5)>[\theta(5)]^{k}=[\theta(ð(\mu, \nu))]^{k},
\end{aligned}
$$

for all $\theta \in \Xi$ and $k \in(0,1)$. Then, Corollary 1 cannot be satisfied.

\section{Set-Valued SU-Type $\Gamma_{\alpha}$-Contraction}

In this section, we prove the existence of fixed point in the class of metric space with respect to a simulation function.

Definition 7. Let $(X, ð)$ be a metric space, $\Lambda$ be a closed subset of $X$, and $\eta$ be a Bianchini-Grandolfi gauge function on $\widehat{E}$. Mapping $T: \Lambda \longrightarrow \mathrm{CB}(X)$ is known as set-valued SUtype $\Gamma_{\alpha}$-contraction, if there exists $\Gamma \in \nabla$ such that for $T(\mu) \cap \Lambda \neq \varnothing$,

$$
\frac{1}{2} \min \{ð(\mu, T(\mu) \cap \Lambda), ð(\nu, T(\nu) \cap \Lambda)\}<ð(\mu, \nu),
$$

which implies that

$$
\Gamma\left[\alpha(\mu, \nu) H_{d}(T(\mu) \cap \Lambda, T(\nu) \cap \Lambda), \eta(\Omega(\mu, \nu))\right] \geq 0,
$$

where

$$
\Omega(\mu, \nu)=\max \left\{ð(\mu, \nu), \text { ठ }(\mu, T(\mu)), ð(\nu, T(\nu)), \frac{\partial(\mu, T(\nu))+ð(\nu, T(\mu))}{2}\right\},
$$

for all $\mu \in \Lambda, \nu \in T(\mu) \cap \Lambda$ with $ð(\mu, \nu) \in \widehat{E}$.

Theorem 3. Let $(X, ð)$ be a complete metric space and $T: \Lambda \longrightarrow \mathrm{CB}(X)$ be a set-valued SU-type $\Gamma_{\alpha}$-contraction such that the following conditions are satisfied:

(a) $T$ is $\alpha$-admissible.

(b) There exists $\mu_{0} \in \Lambda$ with $\partial\left(\mu_{0}, \mu_{1}\right) \in \widehat{E}$ for some $\mu_{1} \in T\left(\mu_{0}\right) \cap \Lambda$ such that $\alpha\left(\mu_{0}, \mu_{1}\right) \geq 1$. Then, there exists an orbit $\left\{\mu_{l}\right\}$ of $T$ in $\Lambda$ and $\vartheta^{*} \in \Lambda$ such that $\lim _{\iota \longrightarrow \infty} \mu_{\iota}=\vartheta^{*}$. In addition, $\vartheta^{*}$ is a fixed point of $T$ if and only if the function $g(\mu):=ð(\mu, T(\mu) \cap \Lambda)$ is T-o.l.s.c at $\vartheta^{*}$.

Proof. By the hypothesis, there exists $\mu_{0} \in \Lambda$ with $ð\left(\mu_{0}, \mu_{1}\right) \in \widehat{E}$ for some $\mu_{1} \in T\left(\mu_{0}\right) \cap \Lambda$ such that $\alpha\left(\mu_{0}, \mu_{1}\right) \geq 1$. On the other hand, we have

$$
\frac{1}{2} \min \left\{\partial\left(\mu_{0}, T\left(\mu_{0}\right) \cap \Lambda\right), \partial\left(\mu_{1}, T\left(\mu_{1}\right) \cap \Lambda\right)\right\}<ð\left(\mu_{0}, \mu_{1}\right) .
$$


In the case that $ð\left(\mu_{0}, \mu_{1}\right)=0, \mu_{0}$ is a fixed point of $T$. Thus, we assume that $ð\left(\mu_{0}, \mu_{1}\right) \neq 0$. Define $\rho=\sigma\left(ð\left(\mu_{0}, \mu_{1}\right)\right)$. From (13), we have $\sigma(r) \geq r$. Hence, $ठ\left(\mu_{0}, \mu_{1}\right) \leq \rho$, and so $\mu_{1} \in \bar{b}\left(\mu_{0}, \rho\right)$. Since $\alpha\left(\mu_{0}, \mu_{1}\right) \geq 1$ and $\partial\left(\mu_{0}, \mu_{1}\right) \in \widehat{E}$, from (76) and (78), it follows that

$$
\begin{array}{r}
0 \leq \Gamma\left[\alpha\left(\mu_{0}, \mu_{1}\right) H_{d}\left(T\left(\mu_{0}\right) \cap \Lambda, T\left(\mu_{1}\right) \cap \Lambda\right), \eta\left(\partial\left(\mu_{0}, \mu_{1}\right)\right)\right] \\
<\eta\left(\Omega\left(\mu_{0}, \mu_{1}\right)\right)-\alpha\left(\mu_{0}, \mu_{1}\right) H_{d}\left(T\left(\mu_{0}\right) \cap \Lambda, T\left(\mu_{1}\right) \cap \Lambda\right),
\end{array}
$$

which implies that

$$
\alpha\left(\mu_{0}, \mu_{1}\right) H_{d}\left(T\left(\mu_{0}\right) \cap \Lambda, T\left(\mu_{1}\right) \cap \Lambda\right)<\eta\left(\Omega\left(\mu_{0}, \mu_{1}\right)\right) .
$$

We can choose $\varepsilon_{1}>0$ such that

$$
\alpha\left(\mu_{0}, \mu_{1}\right) H_{d}\left(T\left(\mu_{0}\right) \cap \Lambda, T\left(\mu_{1}\right) \cap \Lambda\right)+\varepsilon_{1} \leq \eta\left(\Omega\left(\mu_{0}, \mu_{1}\right)\right) .
$$

Thus,

$$
\begin{aligned}
ð\left(\mu_{1}, T\left(\mu_{1}\right) \cap \Lambda\right)+\varepsilon_{1} & \leq H_{d}\left(T\left(\mu_{0}\right) \cap \Lambda, T\left(\mu_{1}\right) \cap \Lambda\right)+\varepsilon_{1} \\
& \leq \alpha\left(\mu_{0}, \mu_{1}\right) H_{d}\left(T\left(\mu_{0}\right) \cap \Lambda, T\left(\mu_{1}\right) \cap \Lambda\right)+\varepsilon_{1} \\
& \leq \eta\left(\Omega\left(\mu_{0}, \mu_{1}\right)\right) .
\end{aligned}
$$

It follows from Lemma 2 that there exists $\mu_{2} \in T\left(\mu_{1}\right) \cap \Lambda$ such that

$$
\text { ð }\left(\mu_{1}, \mu_{2}\right) \leq ð\left(\mu_{1}, T\left(\mu_{1}\right) \cap \Lambda\right)+\varepsilon_{1} .
$$

From fd82(82) and (83), we infer that

$$
\text { ठ }\left(\mu_{1}, \mu_{2}\right) \leq \eta\left(\Omega\left(\mu_{0}, \mu_{1}\right)\right),
$$

where

$$
\begin{aligned}
\Omega\left(\mu_{0}, \mu_{1}\right) & =\max \left\{ð\left(\mu_{0}, \mu_{1}\right), ð\left(\mu_{0}, T\left(\mu_{0}\right)\right), ð\left(\mu_{1}, T\left(\mu_{1}\right)\right), \frac{\partial\left(\mu_{0}, T\left(\mu_{1}\right)\right)+ð\left(\mu_{1}, T\left(\mu_{0}\right)\right)}{2}\right\} \\
& \leq \max \left\{ð\left(\mu_{0}, \mu_{1}\right), ð\left(\mu_{1}, T\left(\mu_{1}\right)\right), \frac{\partial\left(\mu_{0}, T\left(\mu_{1}\right)\right)}{2}\right\} \\
& \leq \max \left\{ð\left(\mu_{0}, \mu_{1}\right), ð\left(\mu_{1}, T\left(\mu_{1}\right)\right)\right\} .
\end{aligned}
$$

We claim that

$$
\text { ð }\left(\mu_{1}, \mu_{2}\right) \leq \eta\left(ð\left(\mu_{0}, \mu_{1}\right)\right) .
$$

Let $\Phi=\max \left\{ð\left(\mu_{0}, \mu_{1}\right), ð\left(\mu_{1}, T\left(\mu_{1}\right)\right)\right\}$. $\Phi=ð\left(\mu_{1}, T\left(\mu_{1}\right)\right)$, we have $\mu_{2} \in T\left(\mu_{1}\right) \cap \Lambda$, so we obtain

$$
\text { ð }\left(\mu_{1}, \mu_{2}\right) \leq \eta\left(ð\left(\mu_{1}, \mu_{2}\right)\right),
$$

which is a contradiction. Thus, we obtain $\Phi=ð\left(\mu_{0}, \mu_{1}\right)$. We assume that $\partial\left(\mu_{1}, \mu_{2}\right) \neq 0$; otherwise, $\mu_{1}$ is a fixed point of $T$. Since $\partial\left(\mu_{1}, \mu_{2}\right) \leq \eta\left(ð\left(\mu_{0}, \mu_{1}\right)\right)<ð\left(\mu_{0}, \mu_{1}\right)$, we deduce that $\partial\left(\mu_{1}, \mu_{2}\right) \in \widehat{E}$. Next, $\mu_{2} \in \bar{b}\left(\mu_{0}, \rho\right)$ because

$$
\begin{aligned}
ð\left(\mu_{0}, \mu_{2}\right) & \leq ð\left(\mu_{0}, \mu_{1}\right)+ð\left(\mu_{1}, \mu_{2}\right) \\
& \leq ð\left(\mu_{0}, \mu_{1}\right)+\eta\left(ð\left(\mu_{0}, \mu_{1}\right)\right) \\
& \leq ð\left(\mu_{0}, \mu_{1}\right)+\sigma\left(\eta\left(ð\left(\mu_{0}, \mu_{1}\right)\right)\right) \\
& =\sigma\left(ð\left(\mu_{0}, \mu_{1}\right)\right)=\rho .
\end{aligned}
$$

Because $T$ is $\alpha$-admissible, $\alpha\left(\mu_{1}, \mu_{2}\right) \geq 1$. Also, since

$$
\frac{1}{2} \min \left\{\partial\left(\mu_{1}, T\left(\mu_{1}\right) \cap \Lambda\right), ð\left(\mu_{2}, T\left(\mu_{2}\right) \cap \Lambda\right)\right\}<ð\left(\mu_{1}, \mu_{2}\right),
$$

from (76), we get

$$
\begin{aligned}
0 & \leq \Gamma\left[\alpha\left(\mu_{1}, \mu_{2}\right) H_{d}\left(T\left(\mu_{1}\right) \cap \Lambda, T\left(\mu_{2}\right) \cap \Lambda\right), \eta\left(\Omega\left(\mu_{1}, \mu_{2}\right)\right)\right] \\
& <\eta\left(\Omega\left(\mu_{1}, \mu_{2}\right)\right)-\alpha\left(\mu_{1}, \mu_{2}\right) H_{d}\left(T\left(\mu_{1}\right) \cap \Lambda, T\left(\mu_{2}\right) \cap \Lambda\right) .
\end{aligned}
$$

This implies that

$$
\alpha\left(\mu_{1}, \mu_{2}\right) H_{d}\left(T\left(\mu_{1}\right) \cap \Lambda, T\left(\mu_{2}\right) \cap \Lambda\right)<\eta\left(\Omega\left(\mu_{1}, \mu_{2}\right)\right) .
$$

Now choose $\varepsilon_{2}>0$ such that

$$
\alpha\left(\mu_{1}, \mu_{2}\right) H_{d}\left(T\left(\mu_{1}\right) \cap \Lambda, T\left(\mu_{2}\right) \cap \Lambda\right)+\varepsilon_{2} \leq \eta\left(\Omega\left(\mu_{1}, \mu_{2}\right)\right) .
$$

Thus,

$$
\begin{aligned}
ð\left(\mu_{2}, T\left(\mu_{2}\right) \cap \Lambda\right)+\varepsilon_{2} \leq & H_{d}\left(T\left(\mu_{1}\right) \cap \Lambda, T\left(\mu_{2}\right) \cap \Lambda\right)+\varepsilon_{2} \\
\leq & \alpha\left(\mu_{1}, \mu_{2}\right) H_{d}\left(T\left(\mu_{1}\right) \cap \Lambda, T\left(\mu_{2}\right) \cap \Lambda\right) \\
& +\varepsilon_{2} \\
\leq & \eta\left(\Omega\left(\mu_{1}, \mu_{2}\right)\right) .
\end{aligned}
$$

It follows from Lemma 2 that there exists $\mu_{3} \in T$, $\left(\mu_{2}\right) \cap \Lambda$ such that

$$
ð\left(\mu_{2}, \mu_{3}\right) \leq ð\left(\mu_{2}, T\left(\mu_{2}\right) \cap \Lambda\right)+\varepsilon_{2} .
$$

From (93) and (94),

$$
ð\left(\mu_{2}, \mu_{3}\right) \leq \eta^{2}\left(\Omega\left(\mu_{0}, \mu_{1}\right)\right),
$$

where 


$$
\begin{aligned}
\Omega\left(\mu_{1}, \mu_{2}\right) & =\max \left\{\partial\left(\mu_{1}, \mu_{2}\right), ð\left(\mu_{1}, T\left(\mu_{1}\right)\right), \partial\left(\mu_{2}, T\left(\mu_{2}\right)\right), \frac{\partial\left(\mu_{1}, T\left(\mu_{2}\right)\right)+ð\left(\mu_{2}, T\left(\mu_{1}\right)\right)}{2}\right\} \\
& \leq \max \left\{\partial\left(\mu_{1}, \mu_{2}\right), ð\left(\mu_{2}, T\left(\mu_{2}\right)\right), \frac{\partial\left(\mu_{1}, T\left(\mu_{2}\right)\right)}{2}\right\} \\
& \leq \max \left\{ð\left(\mu_{1}, \mu_{2}\right), ð\left(\mu_{2}, T\left(\mu_{2}\right)\right)\right\} .
\end{aligned}
$$

We claim that

$$
\text { ð }\left(\mu_{2}, \mu_{3}\right) \leq \eta\left(\partial\left(\mu_{1}, \mu_{2}\right)\right) .
$$

Let $\Phi=\max \left\{\partial\left(\mu_{1}, \mu_{2}\right), ð\left(\mu_{2}, T\left(\mu_{2}\right)\right)\right\}$. If $\Phi=ð\left(\mu_{2}\right.$, $\left.T\left(\mu_{2}\right)\right)$. Since $\mu_{3} \in T\left(\mu_{2}\right) \cap \Lambda$, we have

$$
\text { ð }\left(\mu_{2}, \mu_{3}\right) \leq \eta\left(ð\left(\mu_{2}, \mu_{3}\right)\right),
$$

which is a contradiction. Thus, we have $\Phi=ð\left(\mu_{1}, \mu_{2}\right)$. We assume that $\partial\left(\mu_{2}, \mu_{3}\right) \neq 0$; otherwise, $\mu_{2}$ is a fixed point of $T$. From (95), we have $ð\left(\mu_{2}, \mu_{3}\right)<ð\left(\mu_{1}, \mu_{2}\right)$, and so $ð\left(\mu_{2}, \mu_{3}\right) \in \widehat{E}$. Also, we have $\mu_{3} \in \widehat{b}\left(\mu_{0}, \rho\right)$, since

$$
\begin{aligned}
ð\left(\mu_{0}, \mu_{3}\right) & \leq ð\left(\mu_{0}, \mu_{1}\right)+ð\left(\mu_{1}, \mu_{2}\right)+ð\left(\mu_{2}, \mu_{3}\right) \\
& \leq ð\left(\mu_{0}, \mu_{1}\right)+\eta\left(ð\left(\mu_{0}, \mu_{1}\right)\right)+\eta^{2}\left(ð\left(\mu_{0}, \mu_{1}\right)\right) \\
& \leq \sum_{i=0}^{\infty} \eta^{i}\left(ð\left(\mu_{0}, \mu_{1}\right)\right) \\
& =\sigma\left(ð\left(\mu_{0}, \mu_{1}\right)\right)=\rho .
\end{aligned}
$$

Continuing this setup, we obtain a sequence $\left\{\mu_{l}\right\} \subset \bar{b}\left(\mu_{0}, \rho\right)$ such that $\mu_{t+1} \in T\left(\mu_{l}\right) \cap \Lambda, \mu_{l} \neq \mu_{t+1}$ with $\alpha\left(\mu_{t}, \mu_{t+1}\right) \geq 1$ and $ð\left(\mu_{t}, \mu_{t+1}\right) \in \widehat{E}$ and

$$
\text { ð }\left(\mu_{\iota}, \mu_{\iota+1}\right) \leq \eta^{\imath}\left(ð\left(\mu_{0}, \mu_{1}\right)\right), \quad \text { for all } \iota \in \mathbb{N} .
$$

For $\iota, m \in \mathbb{N}$ with $m>\iota$, by using the triangular inequality and (100), we get

$$
\begin{aligned}
ð\left(\mu_{t}, \mu_{m}\right) \leq & ð\left(\mu_{t}, \mu_{t+1}\right)+ð\left(\mu_{t+1}, \mu_{t+2}\right)+\cdots+ð\left(\mu_{m-1}, \mu_{m}\right) \\
\leq & \eta^{\iota}\left(ð\left(\mu_{0}, \mu_{1}\right)\right)+\eta^{t+1}\left(ð\left(\mu_{0}, \mu_{1}\right)\right)+\cdots \\
& +\eta^{m-1}\left(ð\left(\mu_{0}, \mu_{1}\right)\right) \\
\leq & \sum_{j=\iota}^{\infty} \eta^{j}\left(ð\left(\mu_{0}, \mu_{1}\right)\right)<\infty .
\end{aligned}
$$

To shows that $\left\{\mu_{t}\right\}$ is a Cauchy sequence in $\bar{b}\left(\mu_{0}, \rho\right)$. Since $\bar{b}\left(\mu_{0}, \rho\right)$ is closed in $X$, there exists an $\vartheta^{*} \in \bar{b}\left(\mu_{0}, \rho\right)$ such that $\mu_{t} \longrightarrow \vartheta^{*}$. Note that $\vartheta^{*} \in \Lambda$ because $\mu_{t+1} \in T\left(\mu_{t}\right) \cap \Lambda$. By same argument of Theorem 2, we have

$\frac{1}{2 s} \min \left\{ð\left(\mu_{t}, T\left(\mu_{t}\right) \cap \Lambda\right), ð\left(\mu_{t+1}, T\left(\mu_{t+1}\right) \cap \Lambda\right)\right\}<ð\left(\mu_{t}, \mu_{t+1}\right)$.
Also, we know that $\alpha\left(\mu_{t}, \mu_{t+1}\right) \geq 1$ and $ð\left(\mu_{t}, \mu_{t+1}\right) \in \widehat{E}$ for all $n$. Thus, from (76), we have

$$
\begin{array}{r}
0 \leq \Gamma\left[\alpha\left(\mu_{t}, \mu_{t+1}\right) H_{d}\left(T \mu_{t} \cap \Lambda, T \mu_{t+1} \cap \Lambda\right), \eta\left(\Omega\left(\mu_{t}, \mu_{t+1}\right)\right)\right] \\
<\eta\left(\Omega\left(\mu_{t}, \mu_{t+1}\right)\right)-\alpha\left(\mu_{t}, \mu_{t+1}\right) H_{d}\left(T\left(\mu_{t}\right) \cap \Lambda, T\left(\mu_{t+1}\right) \cap \Lambda\right),
\end{array}
$$

which gives that

$$
\alpha\left(\mu_{\iota}, \mu_{t+1}\right) H_{d}\left(T \mu_{\iota} \cap \Lambda, T \mu_{l+1} \cap \Lambda\right)<\eta\left(\Omega\left(\mu_{\iota}, \mu_{t+1}\right)\right) .
$$

Since $\mu_{t+1} \in T\left(\mu_{t}\right) \cap \Lambda$, from (100), we get

$$
\begin{aligned}
\partial\left(\mu_{t+1}, T\left(\mu_{t+1}\right) \cap \Lambda\right) & \leq \alpha\left(\mu_{t}, \mu_{t+1}\right) H_{d}\left(T\left(\mu_{t}\right) \cap \Lambda, T\left(\mu_{t+1}\right) \cap \Lambda\right) \\
& \leq \eta\left(\Omega\left(\mu_{t}, \mu_{t+1}\right)\right) \\
& <\left(\Omega\left(\mu_{0}, \mu_{1}\right)\right),
\end{aligned}
$$

where

$$
\begin{aligned}
\Omega\left(\mu_{t}, \mu_{t+1}\right) & =\max \left\{\begin{array}{c}
ð\left(\mu_{t}, \mu_{t+1}\right), ð\left(\mu_{t}, T \mu_{t}\right), ð\left(\mu_{t+1}, T \mu_{t+1}\right), \\
\frac{\partial\left(\mu_{t}, T \mu_{t+1}\right)+ð\left(\mu_{t+1}, T \mu_{t}\right)}{2}
\end{array}\right\} \\
& \leq \max \left\{\begin{array}{c}
ð\left(\mu_{t}, \mu_{t+1}\right), ð\left(\mu_{t+1}, \mu_{t+2}\right), \\
\frac{\partial\left(\mu_{t}, \mu_{t+2}\right)}{2}
\end{array}\right\} \\
& \leq \max \left\{ð\left(\mu_{t}, \mu_{t+1}\right), ð\left(\mu_{t+1}, \mu_{t+2}\right)\right\} .
\end{aligned}
$$

We claim that

$$
\begin{aligned}
ð\left(\mu_{t+1}, T\left(\mu_{t+1}\right) \cap \Lambda\right) & \leq \alpha\left(\mu_{t}, \mu_{t+1}\right) H_{d}\left(T\left(\mu_{t}\right) \cap \Lambda, T\left(\mu_{t+1}\right) \cap \Lambda\right) \\
& <\eta\left(\partial\left(\mu_{t}, \mu_{t+1}\right)\right) .
\end{aligned}
$$

Let $\Phi=\max \left\{ð\left(\mu_{t}, \mu_{t+1}\right), ð\left(\mu_{t+1}, \mu_{t+2}\right)\right\} . \quad$ If $\Phi=ð$ $\left(\mu_{t+1}, \mu_{t+2}\right)$. Since $\mu_{t+2} \in T\left(\mu_{t+1}\right) \cap \Lambda$, we have

$$
\begin{aligned}
\text { ð }\left(\mu_{t+1}, T\left(\mu_{t+1}\right) \cap \Lambda\right) & \leq \alpha\left(\mu_{t}, \mu_{t+1}\right) H_{d}\left(T\left(\mu_{t}\right) \cap \Lambda, T\left(\mu_{t+1}\right) \cap \Lambda\right) \\
& <\eta\left(ð\left(\mu_{t+1}, \mu_{t+2}\right)\right),
\end{aligned}
$$

which is a contradiction. Thus, we have $\Phi=ð\left(\mu_{t}, \mu_{t+1}\right)$. Taking limit $\iota \longrightarrow \infty$ in (105), we obtain 


$$
\lim _{\iota \longrightarrow \infty} ð\left(\mu_{t+1}, T\left(\mu_{t+1}\right) \cap \Lambda\right)=0 .
$$

Since $g(\mu)=ð(\mu, T(\mu) \cap \Lambda)$ is T-o.l.s.c at $\vartheta^{*}$, then

$$
\begin{aligned}
\text { ð }\left(\vartheta^{*}, T\left(\vartheta^{*}\right) \cap \Lambda\right) & =g\left(\vartheta^{*}\right) \leq \liminf _{\iota} g\left(\mu_{t+1}\right) \\
& =\liminf _{\iota} \partial\left(\mu_{t+1}, T\left(\mu_{t+1}\right) \cap \Lambda\right)=0 .
\end{aligned}
$$

Since $T \vartheta^{*}$ is closed, $\vartheta^{*} \in T\left(\vartheta^{*}\right)$. Conversely, if $\vartheta^{*}$ is a fixed point of $T$, then $g\left(\vartheta^{*}\right)=0 \leq \liminf _{l} g\left(\mu_{l}\right)$, since $\vartheta^{*} \in \Lambda$.

Taking $\Gamma(r, s)=s-\int_{0}^{r} \varsigma(t) \mathrm{d} t$ for all $r, s \geq 0$, in Theorem 3 , we obtain the following theorem.

Corollary 4. Let $(X, ð)$ be a complete metric space, $\eta$ be a Bianchini-Grandolfi gauge function on an interval $\widehat{E}$, and $T: \Lambda \longrightarrow \mathrm{CB}(X)$ be a given set-valued mapping. If $T \mu \cap \Lambda \neq \varnothing$, then

$$
\frac{1}{2} \min \{ð(\mu, T(\mu) \cap \Lambda), \text { ð }(\nu, T(\nu) \cap \Lambda)\}<ð(\mu, \nu),
$$

which implies that

$$
\int_{0}^{\alpha(\mu, v) H_{d}(T(\mu) \cap \Lambda, T(\nu) \cap \Lambda)} \varsigma(t) \mathrm{d} t \leq \eta(ð(\mu, \nu)),
$$

for all $\mu \in \Lambda, \quad \nu \in T(\mu) \cap \Lambda$, and $ð(\mu, \nu) \in \widehat{E}$, where c: $\mathbb{R}^{+} \longrightarrow \mathbb{R}^{+}$is a function such that $\int_{0}^{\varepsilon} \varsigma(t) \mathrm{d} t$ exists and $\int_{0}^{\varepsilon} \varsigma(t) \mathrm{d} t>\varepsilon$ for all $\varepsilon>0$ such that the following holds:

(a) $T$ is $\alpha$-admissible.

(b) There exists $\mu_{0} \in \Lambda$ with $ð\left(\mu_{0}, \mu_{1}\right) \in \widehat{E}$ for some $\mu_{1} \in T\left(\mu_{0}\right) \cap \Lambda$ such that $\alpha\left(\mu_{0}, \mu_{1}\right) \geq 1$. Then, there exists an orbit $\left\{\mu_{l}\right\}$ of $T$ in $\Lambda$ and $\vartheta^{*} \in \Lambda$ such that $\lim _{l \longrightarrow \infty} \mu_{l}=\vartheta^{*}$. In addition, $\vartheta^{*}$ is a fixed point of $T$ if and only if the function $g(\mu):=ð(\mu, T(\mu) \cap \Lambda)$ is T-o.l.s.c at $\vartheta^{*}$.

Corollary 5. Let $(X, ð)$ be a complete metric space, $\eta$ be a Bianchini-Grandolfi gauge function on an interval $\widehat{E}$, and $T: X \longrightarrow C B(X)$ be a given set-valued mapping. If $\Gamma \in \nabla$ exists, then

$$
\begin{gathered}
\frac{1}{2} \min \{ð(\mu, T(\mu) \cap \Lambda), \text { ठ }(\nu, T(\nu) \cap \Lambda)\}<ð(\mu, \nu) \\
\Rightarrow \Gamma\left[\alpha(\mu, \nu) H_{d}(T(\mu), T(\nu)), \eta(ð(\mu, \nu))\right] \geq 0,
\end{gathered}
$$

for all $\mu \in X, \nu \in T(\mu)$, and $ð(\mu, \nu) \in \widehat{E}$ such that the following holds:

(a) $T$ is $\alpha$-admissible.

(b) There exists $\mu_{0} \in X$ with $ð\left(\mu_{0}, \mu_{1}\right) \in \widehat{E}$ for some $\mu_{1} \in T\left(\mu_{0}\right)$ such that $\alpha\left(\mu_{0}, \mu_{1}\right) \geq 1$. Then, there exists an orbit $\left\{\mu_{\iota}\right\}$ of $T$ in $X$ that converges to the fixed point $\vartheta^{*} \in \mathscr{F}=\left\{\mu \in X: ð\left(\mu, \vartheta^{*}\right) \in \widehat{E}\right\}$ of $T$.

\section{An Application to Dynamical System}

Dynamical system is connected to a multistage operation reduced for solving the following functional equation:

$$
T\left(\mu_{1}\right)=\sup _{\mu_{2} \in H}\left\{h\left(\mu_{1}, \mu_{2}\right)+ð\left(\mu_{1}, \mu_{2}, T\left(l\left(\mu_{1} \mu_{2}\right)\right)\right)\right\} \text { for } \mu_{1} \in \widetilde{\beta},
$$

where

$$
\begin{aligned}
l: \tilde{\beta} \times H & \longrightarrow \widetilde{\beta}, \\
h, h \prime: \tilde{\beta} \times H & \longrightarrow(-\infty, \infty), \\
D, D^{\prime}: \tilde{\beta} \times H \times(-\infty, \infty) & \longrightarrow(-\infty, \infty) .
\end{aligned}
$$

Assume that $\widetilde{G}_{1}$ and $\widetilde{G}_{2}$ are Banach spaces, $\widetilde{\beta} \subset \widetilde{G}_{1}$ is a state space, and $H \subset \widetilde{G}_{2}$ is a decision space. For more details, see [3]. Let $B(\widetilde{\beta})$ signify the set of all bounded real-valued functions on $\widetilde{\beta}$. Choose an arbitrary point $\sigma \in B(\widetilde{\beta})$ defined as $\|\sigma\|=\sup _{r \in \tilde{\beta}}|\sigma(r)| .(B(\tilde{\beta}),\|\|$.$) endowed with the metric$ given by

$$
ð\left(\mu_{1}, \mu_{2}\right)=\sup _{r \in \hat{\beta}}\left|\mu_{1}(r)-\mu_{2}(r)\right|,
$$

for all $\mu_{1}, \mu_{2} \in B(\widetilde{\beta})$, are BS. Define $g: B(\widetilde{\beta}) \longrightarrow B(\widetilde{\beta})$ by

$$
g(\omega)(r)=\max _{t \in H}\{V(r, t, \omega(l(r, t)))+f(r, t)\},
$$

for all $\omega \in B(\widetilde{\beta})$ and $r \in \tilde{\beta}$. Also,

$$
\begin{aligned}
H_{d}\left[g\left(\Phi_{1}\right)(r), g\left(\omega_{2}\right)(r)\right] & =H_{d}\left[V^{x}\left(r, t, \Phi_{1}(l(r, t))\right)+f^{x}(r, t), V^{y}\left(r, t, \Phi_{2}(l(r, t))\right)+f^{y}(r, t)\right] \\
& \leq H_{d}\left[V^{x}\left(r, t, \Phi_{1}(l(r, t))\right), V^{y}\left(r, t, \oplus_{2}(l(r, t))\right)\right] .
\end{aligned}
$$

Consider $\phi: B(\widetilde{\beta}) \longrightarrow B(\widetilde{\beta})$ such that 


$$
\frac{1}{2} \min \left\{\partial\left(\varpi_{1}, \phi\left(\varpi_{1}\right) \cap \Lambda\right), \partial\left(\varpi_{2}, \phi\left(\varpi_{1}\right) \cap \Lambda\right)\right\}<ð\left(\varpi_{1}, \varpi_{2}\right), \varpi_{1}, \varpi_{2} \in B(\tilde{\beta}),
$$

and we have

$$
\begin{aligned}
& \left|V\left(r, t, \oplus_{1}(r)\right)-V\left(r, t, \oplus_{2}(r)\right)\right| \\
& \leq\left[\left[1+\sqrt{T\left(\left|\varpi_{1}(r)-\varpi_{2}(r)\right|\right)}\right]^{\alpha}-1\right]^{2},
\end{aligned}
$$

for all $\varpi_{1}, \Phi_{2} \in B(\tilde{\beta})$, where $r \in \widetilde{\beta}, t \in V$ and $0 \leq \alpha<1$.

Theorem 4. Let $\phi: B(\widetilde{\beta}) \longrightarrow B(\widetilde{\beta})$ be a l.s.c mapping as defined in (117) such that the following conditions are satisfied:

$V$ and $f$ are continuous and bounded.

There exists an orbit $\left\{\varpi_{l}\right\} \in \Lambda$ of $\phi$ and $c^{*} \in \Lambda$ such that $\lim _{\iota \longrightarrow \infty} \varpi_{l}=c^{*}$. $c^{*}$ is a fixed point iff $\phi\left(\varpi_{1}\right)=\circlearrowright\left(\varpi_{1}, T\left(\varpi_{1}\right) \cap \Lambda\right)$ is $\phi$-o.l.s.c at $c^{*}$.

Then, functional (114) possesses a bounded solution.

Proof. Note that $(B(\widetilde{\beta}), \circlearrowright)$ is a complete metric, where $\partial\left(\mu_{1}, \mu_{2}\right)$ is the metric, as defined by (82). There exist $r \in \widetilde{\beta}$, $t_{1}, t_{2} \in V$ and $\psi: B(\widetilde{\beta}) \times B(\widetilde{\beta}) \longrightarrow(0, \infty)$ such that

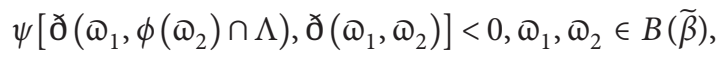

and we have

$$
\begin{aligned}
& H_{d}\left[\phi_{1}\left(\varpi_{1}\right)(r), \phi_{1}\left(\varpi_{2}\right)(r)\right] \leq H_{d}\left[V^{x}\left(r, t, \varpi_{1}(l(r, t))\right), V^{y}\left(r, t, \oplus_{2}(l(r, t))\right)\right] \\
& \leq \max _{r \in \tilde{\beta}}\left\{\max _{t \in \tilde{\beta}}\left|V\left(r, t, \Phi_{1}(l(r, t))\right)-V\left(r, t, \Phi_{2}(l(r, t))\right)\right|\right\} \\
& \leq \max _{r \in \tilde{\beta}}\left\{\max _{t \in \tilde{\beta}}\left\{\left[\left[1+\sqrt{\eta\left(\left|\varpi_{1}(l(r, t))-\varpi_{2}(l(r, t))\right|\right)}\right]^{\alpha}-1\right]^{2}\right\}\right\} \\
& \leq \max _{r \in \tilde{\beta}}\left\{\left[\left[1+\sqrt{\eta\left(\left\|\varpi_{1}-\varpi_{2}\right\|\right)}\right]^{\alpha}-1\right]^{2}\right\} \\
& \leq\left[\left[1+\sqrt{\eta\left(\partial\left(\varpi_{1}, \varpi_{2}\right)\right)}\right]^{\alpha}-1\right]^{2} \text {. }
\end{aligned}
$$

It implies that

$$
H_{d}\left(\phi_{1}\left(\varpi_{1}\right)(r), \phi_{1}\left(\varpi_{2}\right)(r)\right) \leq\left[\left[1+\sqrt{\eta\left(ð\left(\varpi_{1}, \varpi_{2}\right)\right)}\right]^{\alpha}-1\right]^{2} .
$$

Owing to (123),

$$
1+\sqrt{\eta\left(ð\left(ळ_{1}, ळ_{2}\right)\right)} \leq\left[1+\sqrt{\eta\left(ð\left(ळ_{1}, ळ_{2}\right)\right)}\right]^{\alpha} .
$$

By $\theta \in \Xi$ and $\theta(z)=1+\sqrt{z}$ with (124), we obtain

$$
\begin{aligned}
& \theta\left[H_{d} ð\left(\phi_{1}\left(\varpi_{1}\right), \phi_{1}\left(\varpi_{2}\right)\right)\right] \\
& \leq\left[\theta\left(\eta\left(ð\left(\varpi_{1}, \varpi_{2}\right)\right)\right)\right]^{\alpha} \text { for all } \varpi_{1}, \varpi_{2} \in B(\tilde{\beta}) .
\end{aligned}
$$

Furthermore, (1) - (3) are equivalent to $(a)-(b)$ of Theorem 3. So, there exists a fixed point $c^{*}(\in \Lambda)$ in $B(\tilde{\beta})$, which is a bounded solution of functional (117).

4.1. An Application to Integral Inclusion. In this section, we consider the following set-valued integral inclusion:

$$
\varsigma(r) \in \kappa+U \int_{r_{0}}^{r} V(t, \varsigma(t)) V t
$$

where $\kappa \in(-\infty, \infty), U$ is a bounded compact subset of $(-\infty, \infty)$, and $V(t, \varsigma(t))$ is l.s.c. Let $X=C(I)$ be the space of all continuous real-valued function and $C(I)$ is complete w.r.t the metric $ð$, which defined by

$$
ð\left(\mu_{1}, \mu_{2}\right)=\sup _{r \in I}\left|\mu_{1}(r)-\mu_{2}(r)\right| .
$$

Assume that there exists $\phi: B(\widetilde{\beta}) \longrightarrow B(\widetilde{\beta})$ and $V:(-\infty, \infty) \times(-\infty, \infty) \longrightarrow(-\infty, \infty)$ is continuous on

$$
R=\left\{(r, \varsigma):\left|r-r_{0}\right| \leq\left[\frac{1}{\alpha_{1}}\right]^{1 / 2} \text { and }|\varsigma-\kappa| \leq \frac{1}{2 \alpha_{2}}\right\},
$$

such that for

$$
\frac{1}{2} \min \left\{ð\left(\mu_{1}, \phi\left(\mu_{1}\right) \cap \Lambda\right), ð\left(\mu_{2}, \phi\left(\mu_{2}\right) \cap \Lambda\right)\right\}<ð\left(\varpi_{1}, \varpi_{2}\right),
$$

$$
\mu_{1}, \mu_{2} \geq 0,
$$

we have

$$
\left|V\left(r, \varsigma_{1}(r)\right)-V\left(r, \varsigma_{2}(r)\right)\right| \leq e^{\sqrt{\alpha_{1} / \alpha_{2}\left|\varsigma_{1}(r)-\varsigma_{2}(r)\right|^{\alpha}}},
$$

where $\alpha_{2}=\max _{\bar{u} \in U}|\bar{u}|, 0<\alpha_{1} \leq \alpha_{2}$, and $0 \leq \alpha<1$. 


$$
|V(t, \varsigma)|<\frac{1}{2 \alpha_{1}}\left[\frac{1}{\alpha_{1}}\right]^{1 / 2} .
$$

Moreover, let $\check{C}=\left\{\varsigma \in C(I): ð(\varsigma, \kappa) \leq 1 / 2 \alpha_{2}\right\}$ be a closed subspace of $C(I)$ and the operator $\phi$ be defined by

$$
\phi(\varsigma(r)) \in \kappa+U \int_{r_{0}}^{r} V(t, \varsigma(t)) \mathrm{d} t .
$$

Set $V_{x}(r)=\int_{r_{0}}^{r} V(t, \varsigma(t)) \mathrm{d} t$. Note that

$$
\begin{aligned}
H_{d}\left[\phi\left(\varsigma_{1}(r)\right), \phi\left(\varsigma_{2}(r)\right)\right]= & H_{d}\left[\kappa+U V_{x}(r), \kappa+U V_{y}(r)\right] \\
& \leq H_{d}\left[U V_{x}(r), U V_{y}(r)\right] \\
= & \max \left\{\max _{\bar{a} \in U V_{x}(r)} ð\left(\bar{a}, U V_{y}(r)\right), \max _{\bar{b} \in U V_{y}(r)} ð\left(\bar{b}, U V_{x}(r)\right)\right\} .
\end{aligned}
$$

Consider

$$
\begin{aligned}
& \max _{\bar{a} \in U V_{x}(r)} \text { ð(}\left(\bar{a}, U V_{y}(r)\right)=\max _{\bar{a} \in U V_{x}(r)} \min _{\bar{b} \in U V_{y}(r)} \text { ð }(\bar{a}, \bar{b}) \\
& =\max _{\bar{u} \in U} \min _{\bar{v} \in U} ð\left(\bar{u} V\left(r, \varsigma_{1}(r)\right), \bar{v} V\left(r, \varsigma_{2}(r)\right)\right) \\
& =\max _{\bar{u} \in U} \min _{\bar{v} \in U} \sup _{r \in I}\left|\bar{u} V\left(r, \varsigma_{1}(r)\right)-\bar{v} V\left(r, \varsigma_{2}(r)\right)\right| \\
& \leq \max _{\bar{u} \in U} \min _{\bar{v} \in U} \sup _{r \in I}\left[\left|\bar{u} V\left(r, \varsigma_{2}(r)\right)-\bar{v} V\left(r, \varsigma_{2}(r)\right)\right|+\left|\bar{u} V\left(r, \varsigma_{2}(r)\right)-\bar{u} V\left(r, \varsigma_{1}(r)\right)\right|\right] \\
& \leq \max _{\bar{u} \in U} \min _{\bar{v} \in U}\left[|\bar{u}| \sup _{r \in I}\left|V\left(r, \varsigma_{2}(r)\right)-V\left(r, \varsigma_{1}(r)\right)\right|+|\bar{u}-\bar{v}| \sup _{r \in I}\left|V\left(r, \varsigma_{2}(r)\right)\right|\right] \\
& =\max _{\bar{u} \in U}|\bar{u}| \sup _{r \in I}\left|V\left(r, \varsigma_{2}(r)\right)-V\left(r, \varsigma_{1}(r)\right)\right| \\
& =\alpha_{2} \sup _{r \in I}\left|V\left(r, \varsigma_{2}(r)\right)-V\left(r, \varsigma_{1}(r)\right)\right| \text {. }
\end{aligned}
$$

This implies that

$$
\max _{\bar{a} \in U V_{x}(r)} V\left(\bar{a}, U V_{y}(r)\right) \leq \alpha_{2} \sup _{r \in I}\left|V\left(r, \varsigma_{2}(r)\right)-V\left(r, \varsigma_{1}(r)\right)\right| .
$$

Theorem 5. Let $X=C(I)$ and $\phi:(\check{C}, d) \longrightarrow\left(D(\check{C}), H_{d}\right)$ be a l.s.c mapping. Suppose that the following assumptions hold:

(i) $\phi$ is defined for all $\varsigma \in \check{C}$.

(ii) $\phi(\varsigma(r))$ is a CS of $\check{C}$ for all $\varsigma \in \check{C}$.

Then, owing to (127)-(135), integral (126) has a solution on $I$.

Proof. Let $\varkappa \in I$. Then, $\left|\varkappa-r_{0}\right| \leq\left[1 / \alpha_{1}\right]^{1 / 2}$. Hence, we have $|\varsigma(\varkappa)-\kappa| \leq 1 / 2 \alpha_{1}$. If $(\varkappa, \varsigma(x)) \in(-\infty, \infty)$, the integral equation in (132) exists. Since $\kappa \in(-\infty, \infty)$ is continuous, $\varkappa$ is defined for all $\varkappa \in \check{C}$. Next, let $\vartheta(r) \in \phi(\varsigma(r))$. Then, $\vartheta(r)=\kappa+\bar{u} V_{x}(r)$ for $\bar{u} \in U:$

$$
\begin{aligned}
|\vartheta(r)-\kappa| & =\left|\bar{u} V_{x}(r)\right|=|\bar{u}|\left|V_{x}(r)\right| \\
& \leq \alpha_{2} \int_{r_{0}}^{r}|V(t, \varsigma(t)) \mathrm{d} t| \\
& \leq \alpha_{2} \int_{r_{0}}^{r}|V(t, \varsigma(t))| \mathrm{d} t \\
& <\alpha_{2}\left|r-r_{0}\right| \frac{1}{2 \alpha_{2}}\left[\frac{1}{\alpha_{1}}\right]^{1 / 2} \\
& \leq \frac{1}{2 \alpha_{1}} .
\end{aligned}
$$

Thus, $|\vartheta(r)-\kappa| \leq 1 / 2 \alpha_{1}$ for each $\vartheta(r) \in \phi(\varsigma(r))$. So, $\phi(\varsigma(r))$ is a subset of $\check{C}$. Now, let $\left\{\varsigma_{i}\right\} \subset \phi(\varsigma(r))$; then, $\varsigma=$ $\kappa+\overline{u_{i}} V_{x}(r)$ for $\overline{u_{i}} \in U$. Since $U$ is compact, there exists subsequence $\widehat{u_{i^{*}}} \in \widehat{u_{i}}$ such that $\left\{\widehat{u_{i^{*}}}\right\}$ is convergent to $\bar{u} \in U$. Let $\widehat{u}=\kappa+\widehat{u} V_{x}(r)$; then, 


$$
\begin{aligned}
d\left(\widehat{u_{i^{*}}}, \widehat{u}\right) & =\sup _{r \in I}\left(\left|\widehat{u_{i^{*}}}-\widehat{u}\right|\left|V_{x}(r)\right|\right) \\
& \leq\left|\widehat{u_{i^{*}}}-t \widehat{u}\right| \sup _{r \in I}\left|V_{x}(r)\right| \longrightarrow 0, \text { as } i^{*} \longrightarrow \infty .
\end{aligned}
$$

Hence, $\phi(\varsigma(r))$ is a CS of $\check{C}$ for all $\varsigma \in \check{C}$. Next,

$$
\begin{aligned}
\left|V\left(r, \varsigma_{1}(r)\right)-V\left(r, \varsigma_{2}(r)\right)\right| & \leq \int_{r_{0}}^{r}\left|V\left(t, \varsigma_{1}(t)\right)-V\left(t, \varsigma_{2}(t)\right)\right| \mathrm{d} t \\
& \leq e^{\int_{r_{0}}^{r} \sqrt{\alpha_{1} / \alpha_{2}\left|\varsigma_{1}(t)-\varsigma_{2}(t)\right|^{\alpha} \mathrm{d} t}} \\
& =e^{\sqrt{\alpha_{1} / \alpha_{2}} \int_{r_{0}}^{r} \sqrt{\left|\varsigma_{1}(t)-\varsigma_{2}(t)\right|^{\alpha} \mathrm{d} t}} \\
& \leq e^{\sqrt{\alpha_{1} / \alpha_{2}}\left(\sup _{r \in I}\left|\varsigma_{1}(t)-\varsigma_{2}(t)\right|^{\alpha}\right)^{1 / 2} \int_{r_{0}}^{r}} \mathrm{~d} t \\
& =e^{\sqrt{\alpha_{1} / \alpha_{2}}\left|r-r_{0}\right|\left(\sup _{r \in I}\left|\varsigma_{1}(t)-\varsigma_{2}(t)\right|^{\alpha}\right)^{1 / 2}} \\
& \leq e^{\sqrt{\alpha_{1} / \alpha_{2}}\left(1 / \alpha_{2}\right)^{1 / 2}\left(\partial\left(\varsigma_{1}(t)-\varsigma_{2}(t)\right)^{\alpha}\right)^{1 / 2}} \\
& =e^{\sqrt{1 / \alpha_{2} \partial\left(\varsigma_{1}(t)-\varsigma_{2}(t)^{\alpha}\right)}} .
\end{aligned}
$$

It yields that

$$
\left|V\left(r, \varsigma_{1}(r)\right)-V\left(r, \varsigma_{2}(r)\right)\right| \leq e^{\sqrt{\alpha_{1} / \alpha_{2}}\left(1 / \alpha_{2}\right)^{1 / 2}\left(ð\left(\varsigma_{1}(t)-\varsigma_{2}(t)\right)^{\alpha}\right)^{1 / 2}} .
$$

By $\theta \in \Xi, \theta(z)=e^{\sqrt{z}}$ and $\eta(r)=1 / \alpha_{2} r$ with (139), we get

$$
\theta\left[H_{d} \partial\left(\phi\left(\varpi_{1}\right), \phi\left(\varpi_{2}\right)\right)\right] \leq\left[\theta\left(\eta\left(ð\left(\varpi_{1}, \varpi_{2}\right)\right)\right)\right]^{\alpha} .
$$

Furthermore, (i)-(ii) are equivalent to $(a)-(b)$ of

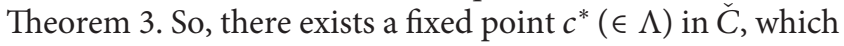
is a bounded solution of (126).

\section{Conclusion}

The paper deals with the set-valued fixed point theorems satisfying SU-type contraction via Bianchini-Grandolfi gauge function in the context of metric spaces. Within this framework, we introduced two related fixed point results in metric space. An extensive set of nontrivial example is given to justify our claim. Also, we have proven the existence theorem for the system of functional equation and integral inclusion.

\section{Data Availability}

The data used to support the findings of this study are included within the article.

\section{Conflicts of Interest}

The authors declare that there are no conflicts of interest.

\section{Authors' Contributions}

All authors contributed equally to the writing of this paper. All authors read and approved the final manuscript.

\section{Acknowledgments}

The authors are thankful to the Deanship, King Abdulaziz University, Jeddah, KSA, for funding this research.

\section{References}

[1] M. Jleli and B. Samet, "A new generalization of the Banach contraction principle," Journal of Inequalities and Applications, vol. 38, 2014.

[2] F. Khojasteh, S. Shukla, and S. Radenović, "A new approach to the study of fixed point theorems via simulation functions," Filomat, vol. 29, pp. 1189-1194, 2015.

[3] A. Ali, F. Uddin, M. Arshad, and M. Rashid, "Hybrid fixed point results via generalized dynamic process for F-HRS type contractions with application," Physica A: Statistical Mechanics and Its Applications, vol. 538, Article ID 122669, 2020.

[4] M. Abbas, H. qbal, and A. etrusel, "Fixed points for multivalued Suzuki type $(\theta, R)$-contraction mapping with applications," Journal Function Spaces, vol. 13, 2019.

[5] B. Sam, "Set-valued contraction mappings," Pacific Journal of Mathematics, vol. 10, pp. 475-488, 1969.

[6] F. Vetro, "A generalization of Nadler fixed point theorem," Carpathian Journal of Mathematics, vol. 31, pp. 403-410, 2015.

[7] P. D. Proinov, "A generalization of the Banach contraction principle with high order of convergence of successive approximations," Nonlinear Analysis: Theory, Methods \& Applications, vol. 67, no. 8, pp. 2361-2369, 2007. 